\title{
Detection and Prevention of Forward Head Posture with Body Sensor Networks
}

\author{
A Thesis \\ Presented to \\ the faculty of the School of Engineering and Applied Science \\ University of Virginia
}

In partial fulfillment

of the requirements for the degree

Master of Science

by

Taeyoung Kim

May

2012 


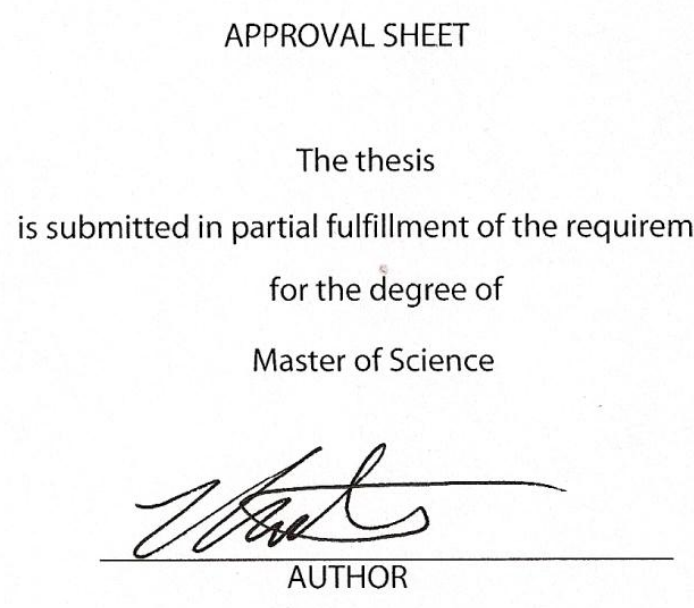

The thesis has been read and approved by the examining committee:

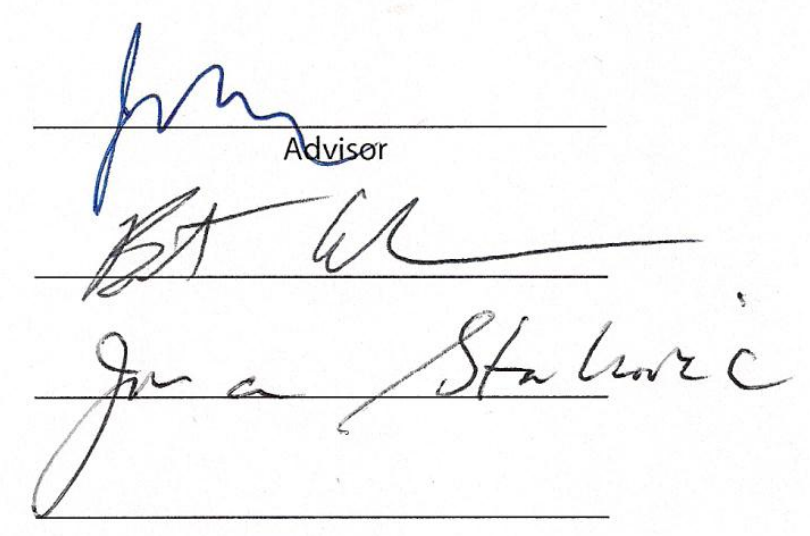

Accepted for the School of Engineering and Applied Science:

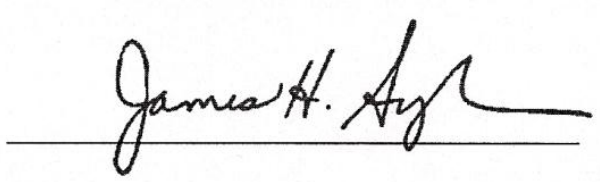

Dean, School of Engineering and Applied Science

May 


\section{Acknowledgements}

This thesis was a joint effort by the support of colleagues, friends, and family. I would like to thank all those who have supported me, and my particular thanks to the following:

My advisor, Dr. John Lach, has been always leading me onto the right path of being a better researcher. Moreover, he has counseled and supported me with regard to my personal and family issues as a strong mentor for three years. He will be ever in my thoughts.

I also give my appreciation to my friends and colleagues. I wish to extend special acknowledgement to my INERTIA teammates: Adam Barth, Jeff Brantly, Shanshan Chen, Sam Ridenour, Italo Armenti, Philip Asare, and Juliana Su.

I would like to sincerely thank my Master's thesis committee, Dr. Benton H. Calhoun and Dr. John A. Stankovic, for their efforts and feedback on this thesis

Lastly, I thank my wife, son, and my unborn child for the sacrifices they have made for me to successfully finish my degree. 


\section{Abstract}

Forward Head Posture (FHP) is a common musculoskeletal disorder correlated with neck pain that affects a large percentage of the population. Research has shown that providing feedback for posture correction can help combat FHP and reduce the associated neck pain. Currently, the methods for head posture detection have limited effectiveness due to the immobility and invasiveness of the monitoring systems and/or the inaccurate results they produce.

This thesis offers a continuous, non-invasive solution for assessing craniovertebral (CV) angle for FHP detection using a wireless inertial body sensor platform. The results obtained are validated against a conventional in-clinic method, the Electronics Head Posture Instrument (EHPI), demonstrating the possibility of pervasive detection of FHP. In addition, a real-time bio-feedback mechanism for postural correction and prevention is presented, and results from a pilot study show its effectiveness in reducing the amount of time subjects spend in FHP. 
To Yunji, Jihu, the unborn baby, and for anyone who suffers from FHP 


\section{Table of Contents}

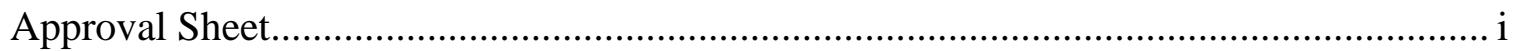

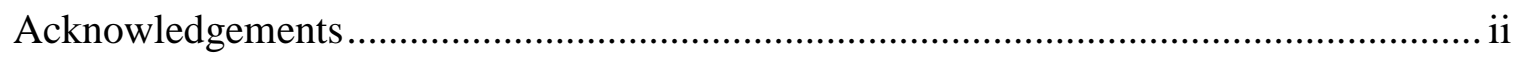

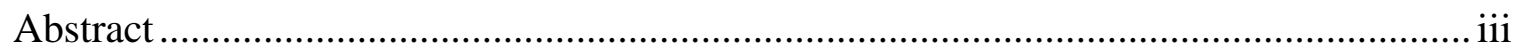

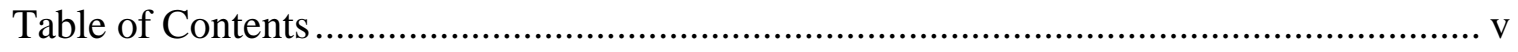

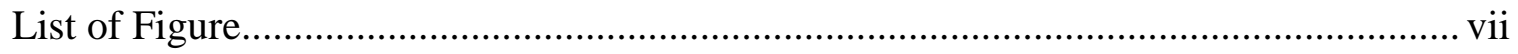

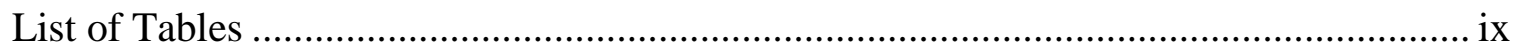

Chapter $1 \quad$ : Introduction.................................................................................. 1

Chapter 2 Current Technology ......................................................................... 5

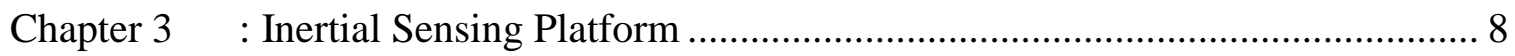

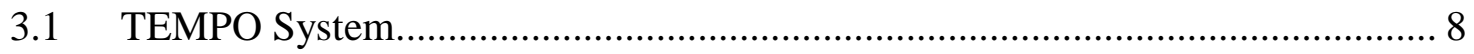

S.2 Sensor Calibration ........................................................................... 11

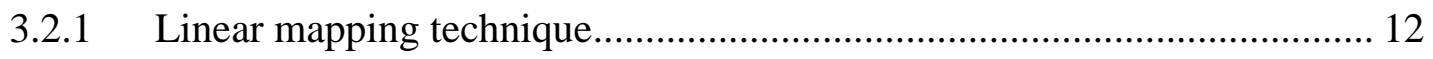

3.2.2 Mathematical estimation technique ................................................. 12

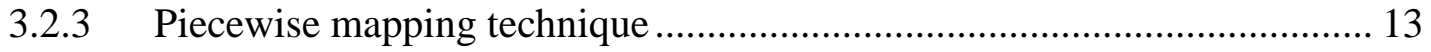




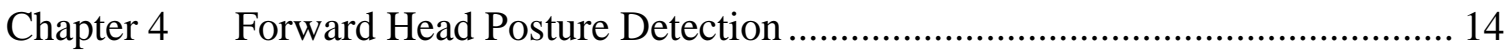

4.1 Number and Location of Sensors .................................................................... 15

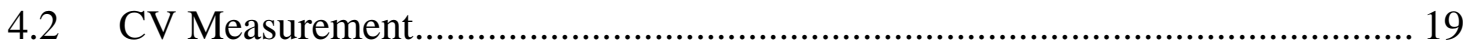

4.3 Real-time Monitoring System ................................................................. 21

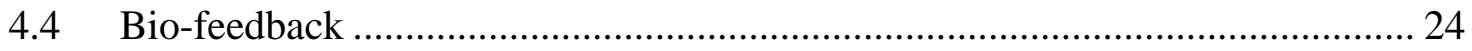

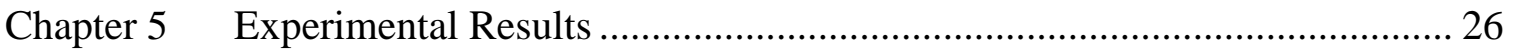

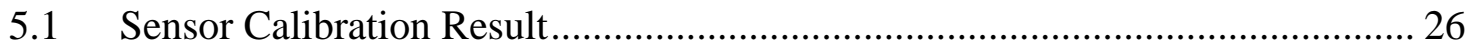

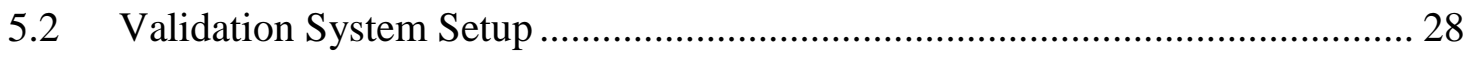

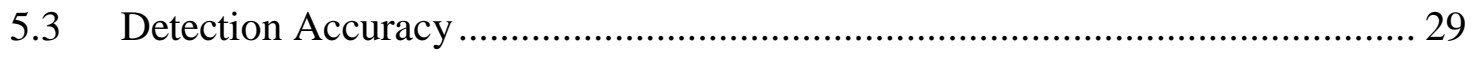

5.4 Evaluation of the Bio-feedback .................................................................. 32

Chapter 6 Conclusion and Future Work .......................................................... 35

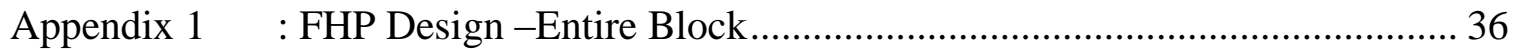

Appendix 2 Conversion/Window/LP .......................................................... 39

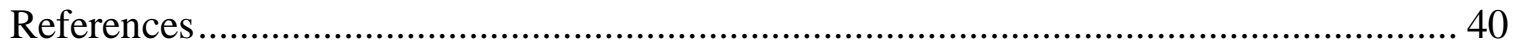

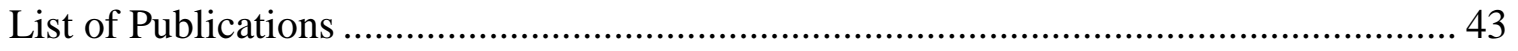




\section{List of Figure}

Figure 1 Normal Posture (Left) and Forward Head Posture (Right) .............................. 2

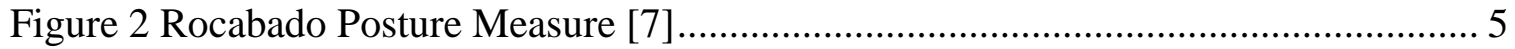

Figure 3 EHPI Instrument for CV Angle Assessment [18] ...................................... 6

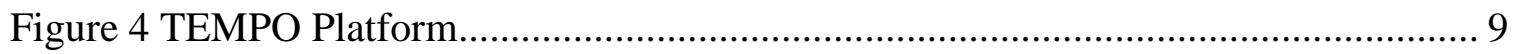

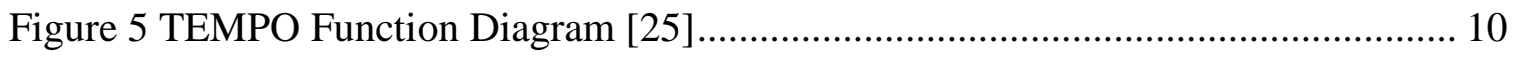

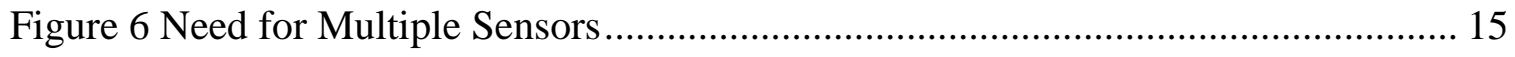

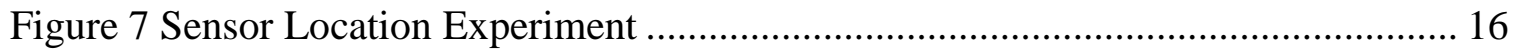

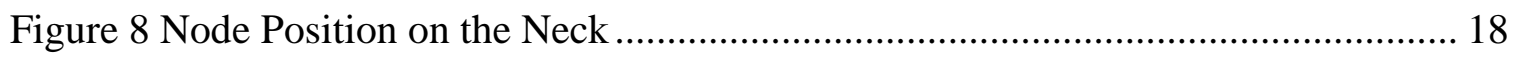

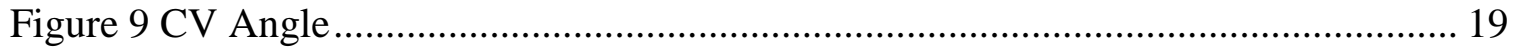

Figure 10 FHP Monitoring System Functions .................................................... 21

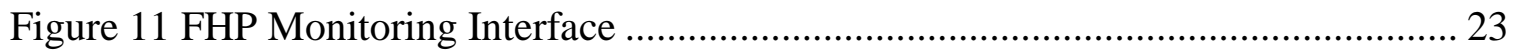

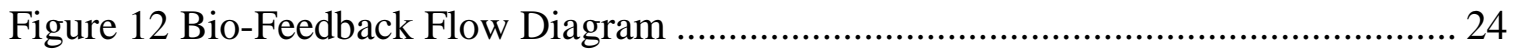

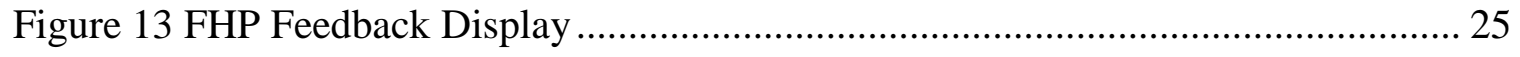

Figure 14 Calibration and Measurement Instrument .............................................. 26

Figure 15 Validation System Setup with EHPI for Initial Posture ............................... 28

Figure 16 Validation with Image Processing for Various Postures ............................... 29 
Figure 17 Effectiveness of Bio-feedback with FHP Monitoring ................................... 33 


\section{List of Tables}

Table 1 Correlation Coefficient of Nodes Position...................................................... 17

Table 2 The Measurement for Node Position on the Neck ........................................ 18

Table 3 Accuracy Analysis of 1-axis Calibrated Accelerometer.................................. 27

Table 4 CV Measurement for Various Postures ...................................................... 30

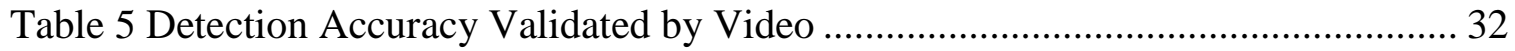




\section{Chapter 1 : Introduction}

Inertial Body Sensor Networks (BSNs) have emerged as an accurate yet non-invasive method for detecting and assessing the movements of a human body. Posture monitoring based on inertial BSN technology shows a promising future in preventing a common musculoskeletal disorder known as Forward Head Posture (FHP). Unlike traditional methods where a patient must remain immobile while a doctor diagnoses the disorder, inertial BSNs offer a less invasive and more cost-effective alternative where a patient's FHP can be immediately detected via continuous monitoring and corrected using realtime bio-feedback.

FHP afflicts a large percentage of the population (22.2\% of adults and $6.9 \%$ of children) [1][3][4]. People who suffer from FHP are accustomed to positioning the head in front of the body, which places great strain on the cervical spine and can ultimately be quite painful [5]. FHP has also been shown to be related to temporomandibular disorders [6], cervicogenic headache, thoracic outlet syndrome, cervical spondylogenic changes [7][8][9], loss of proper bowel function and reduced vital capacity [10]. It is also associated with shortening of the posterior cervical extensor muscles and with tightening of the anterior cervical muscles [11]. 

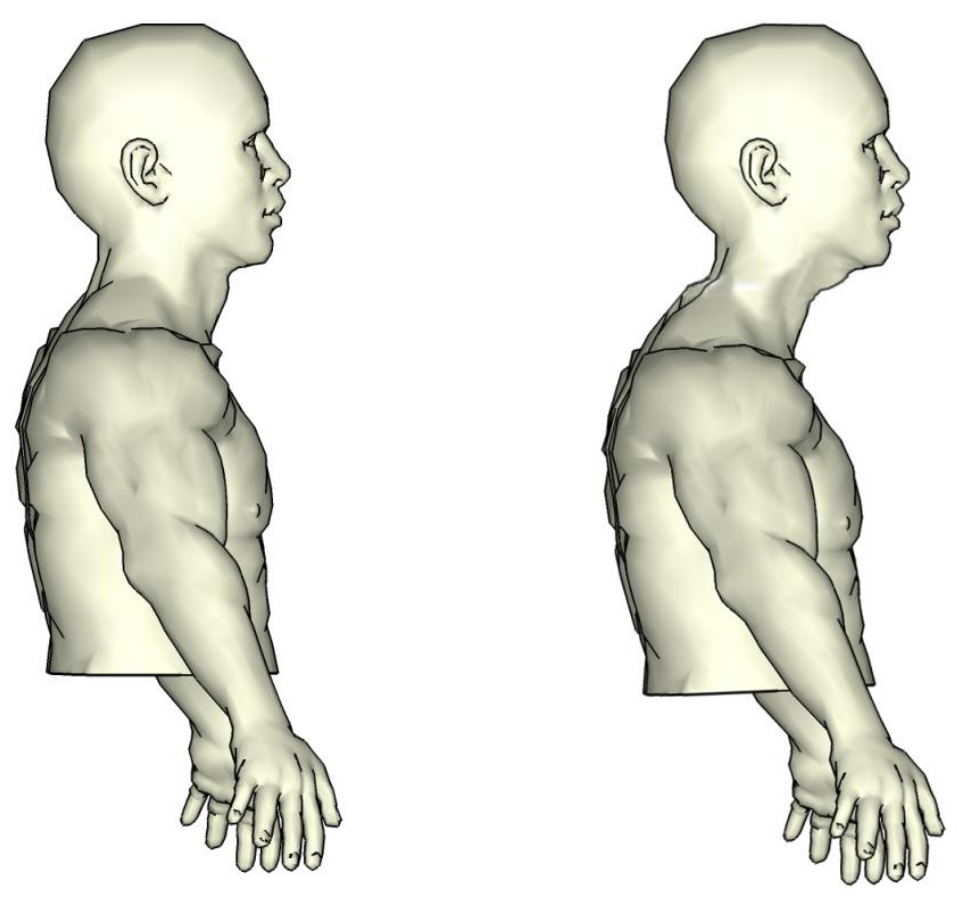

Figure 1 Normal Posture (Left) and Forward Head Posture (Right)

Although the consensus in the medical field is that FHP is a disorder caused by improper posture, there is no consensus regarding its diagnosis [7][12][14]. Originally, FHP was described as the position in which the head rests anterior to the anatomic points of reference, as seen in Figure 1. The experts who study FHP tend to describe FHP as a condition in which a protruding head with an extended upper and protracted lower cervical joint [12][13][14], or an extension of the back part of the skull on the top cervical vertebra occurred with an increasing of the upper cervical lordosis [5][9][15]. Other experts only state that FHP is a condition associated with an extension of the head in relation to the cervical column without reference to the associated deviant head posture [16][17].

A common contraption that is used to evaluate FHP is the Electronic Head Posture Instrument (EHPI) [18], which uses an inclinometer for measuring of craniovertebral (CV) 
angle. However, the conventional instruments have limited effectiveness since they can only be used in clinical settings and cannot provide continuous monitoring for selfmonitoring and auto-correction [19]. An effective instrument would offer a low-cost and user-friendly system that is designed to monitor a patient's posture and alerts the patient when the patient engages in an improper posture that causes FHP. Since FHP most often occurs while a person is sitting at a desk reading, using a computer, etc. for an extended period of time, a device that is portable enough to be used in an office or home environment to continuously monitor a person's posture - and provide timely biofeedback - is highly desirable.

This thesis proposes a novel solution for FHP detection and real-time prevention by assessing $\mathrm{CV}$ angle using inertial BSNs. In order to measure the $\mathrm{CV}$ angle, two accelerometers are used to detect the raw angles of head and neck of human body with the TEMPO BSN platform [24]. TEMPO system can send data to the FHP monitoring system to calculate the CV angle. The FHP monitoring system uses the low pass filter to reduce the small human movement noise. In order to provide effective bio-feedback, a sliding window with a standard deviation threshold is used to detect static periods. Once the CV angle is below the clinically acknowledged angle for FHP, a bio-feedback alarm will alert the user of its improper posture. The effectiveness of this bio-feedback is tested on four human subjects.

This thesis describes two key contributions to the BSN area:

1. This work presents a general approach for applying an inertial BSN technology to improve the posture of people afflicted with FHP. This thesis first discusses 
the sensor calibration method developed to increase the accuracy of the angle measurement. Next, the thesis determines the correct sensor placement and position to give an accurate reading. Finally, this paper examines the $\mathrm{CV}$ angle detection algorithm that is designed to alert the users who may not be aware of their improper posture.

2. This work explores the issue of managing and controlling real-time biofeedback based on continuous monitoring, which is an underexplored area in BSN research. The effectiveness of this bio-feedback was evaluated in a small human subject study focused on reducing FHP for people working on a computer at a desk. In addition, this research emphasizes the trade-off between compliance and prompt/regular bio-feedback to maximize the effectiveness of the system [20].

This thesis is organized as follows. Chapter 2 discusses the historical applications of CV measurement and how it can be adapted to treat FHP. Chapter 3 presents the inertial sensor calibration method for inertial BSN application and a brief look at the BSN platform used in this research. Chapter 4 describes the posture monitoring system equipped with the bio-feedback mechanism that enables detection of FHP. Chapter 5 evaluates the system's accuracy in detecting and providing bio-feedback to the user and the resulting reduction in the subjects' time spent in FHP. Finally, Chapter 6 discusses the conclusions of this work and the future work for this and related technology. 


\section{Chapter 2 Current Technology}

The T-shaped Rocabado Posture Gauge, shown in Figure 2, is the first instrument used to evaluate FHP in 1983 [7]. The instrument measures the horizontal line from the outmost thoracic spine to the innermost cervical spine of a patient in a standing position. The measured distances were used to determine whether a patient suffered from FHP [21].
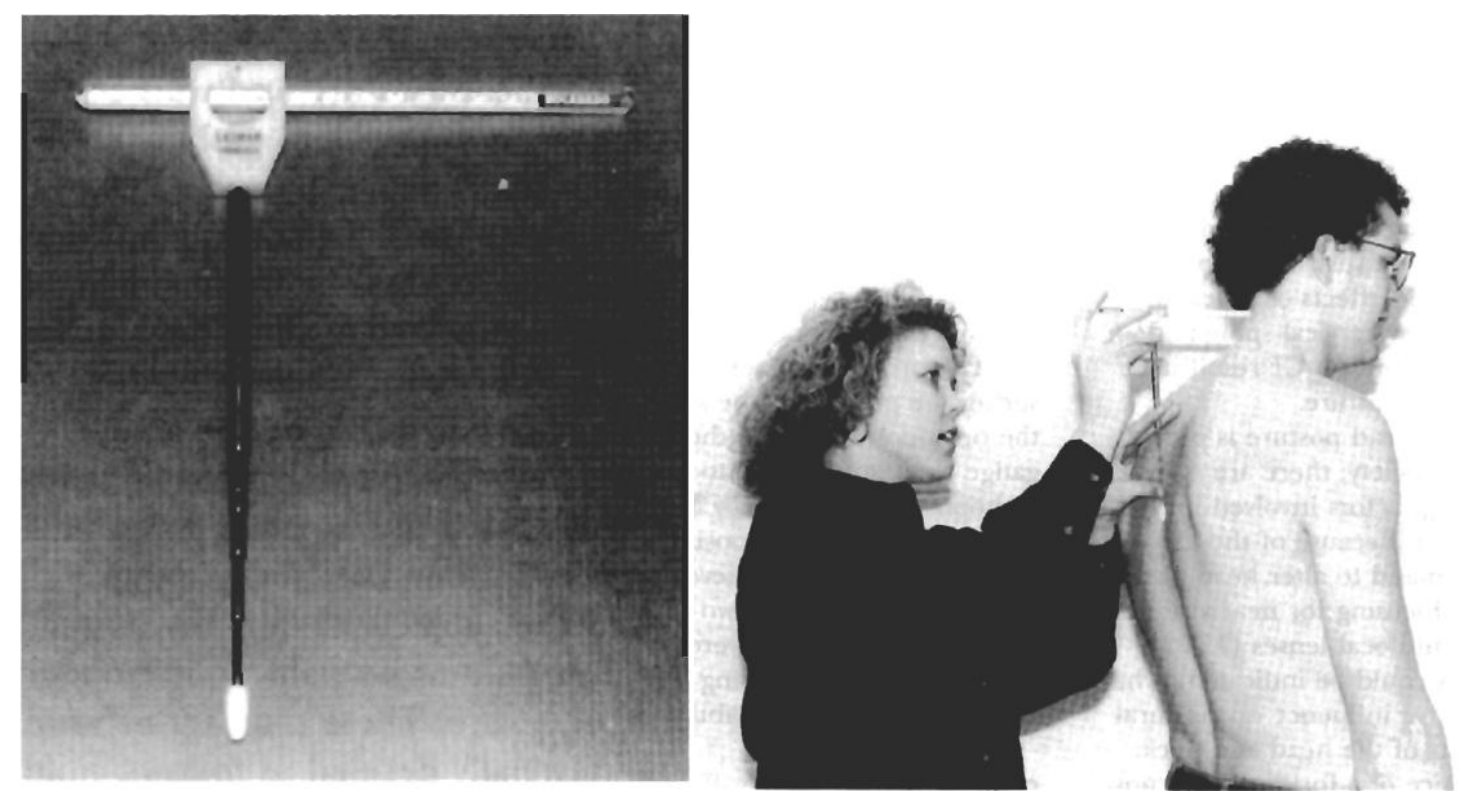

Figure 2 Rocabado Posture Measure [7]

Head posture diagnosis is also determined by the posture imagining and plumb line [22].

However, the accuracy of this method is questionable due to its subjective nature. For 
example, the measurement of the degree of forward head posture can vary with the viewing angle of the examiner in relation to the patient. Furthermore, photographic imaging does not offer a better solution because of the delayed time required to obtain an accurate assessment [22].

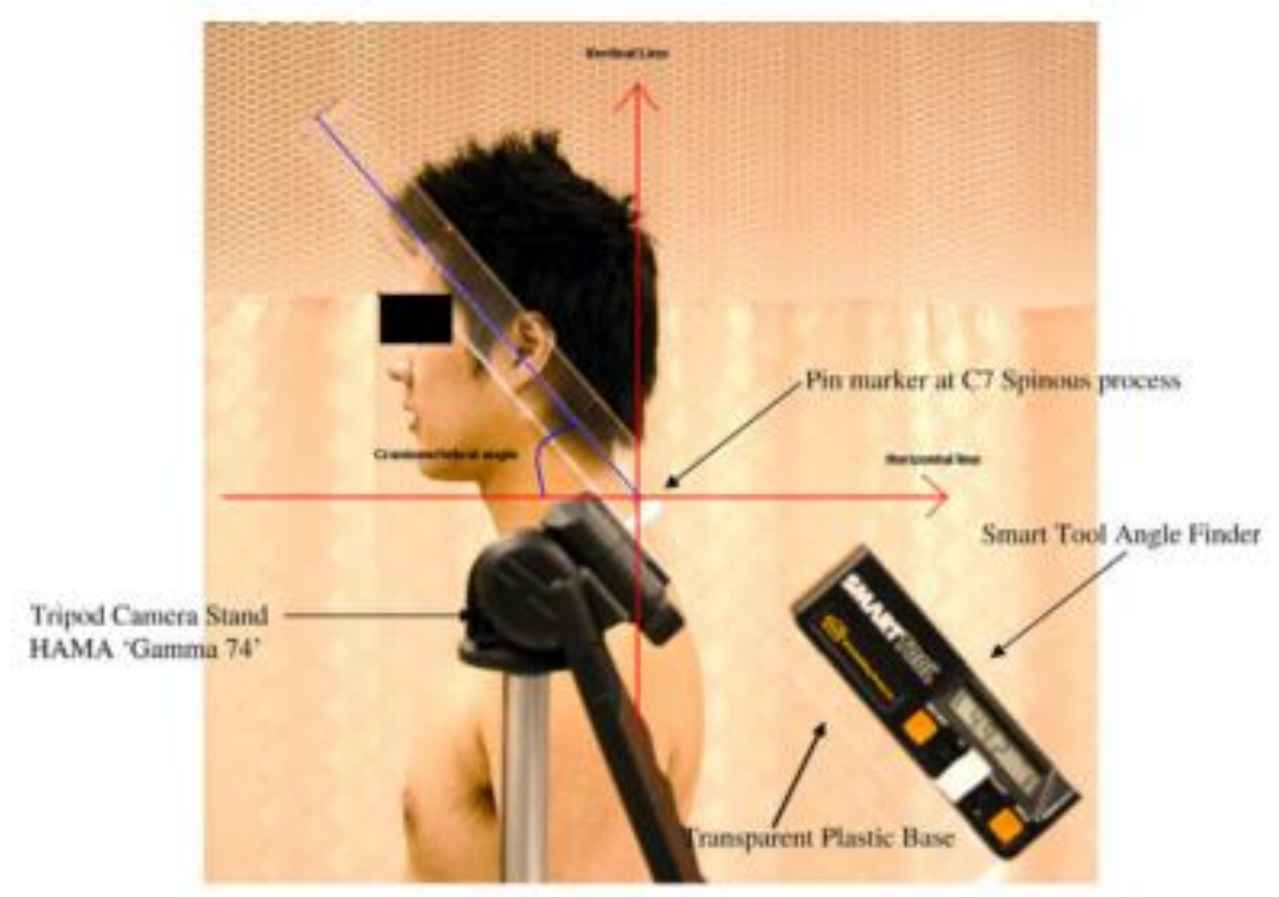

Figure 3 EHPI Instrument for CV Angle Assessment [18]

Another method of evaluating head posture is through measuring the craniovertebral (CV) angle. This is the angle between a horizontal line through the spinous process of the seventh cervical vertebra (C7) and a line from spinous process of $\mathrm{C} 7$ through the tragus of the ear [22].

The latest tool that is used to measure the CV angle is the Electronic Head Posture Instrument (EHPI) [18], as shown in Figure 3. The reliability of the EHPI is high in measuring the $\mathrm{CV}$ angle of individuals with normal posture and those who suffer from 
FHP. The CV angles measured on patients with chronic pain were significantly smaller than the health controls [18].

Despite EHPI's accuracy, it is not without limitations. First, this machine can only be used in clinics by professionals who diagnose FHP. It is not designed to be a tool to prevent FHP, but rather just detect it. Second, FHP occurs while people are engaged in normal daily activities such as watching TV, reading a book, working on a computer, and sleeping in their bed. Therefore, a device featuring a continuous monitoring system, which can alert the user of improper posture, can be an effective tool to treat FHP. 


\section{Chapter 3 : Inertial Sensing Platform}

In this Chapter, the inertial sensing platform - providing 3-axes of acceleration with respect to gravity - that is used for FHP monitoring system will be described, including the techniques developed for sensor calibration.

\subsection{TEMPO System}

TEMPO is an inertial sensing device that provides six degrees of freedom motion capture in a device roughly the size of a watch. The device uses accelerometers to measure linear acceleration in all three axes, as well as gyroscopes to measure rotational rate in all three planes at sampling rates as high as $128 \mathrm{~Hz}$. The acceleration is measured with Freescale MMA7331 in this system. The accelerometer can measure up to $+-12 \mathrm{~g}$ for the each axis. The sensor outputs are analog and are filtered with simple passive filters using resistors and capacitors. The orientation of the sensors on the devices is described in Figure 4. The data is than wirelessly transmitted via Bluetooth to an aggregator such as a smartphone, a laptop, or other mobile devices [24]. 

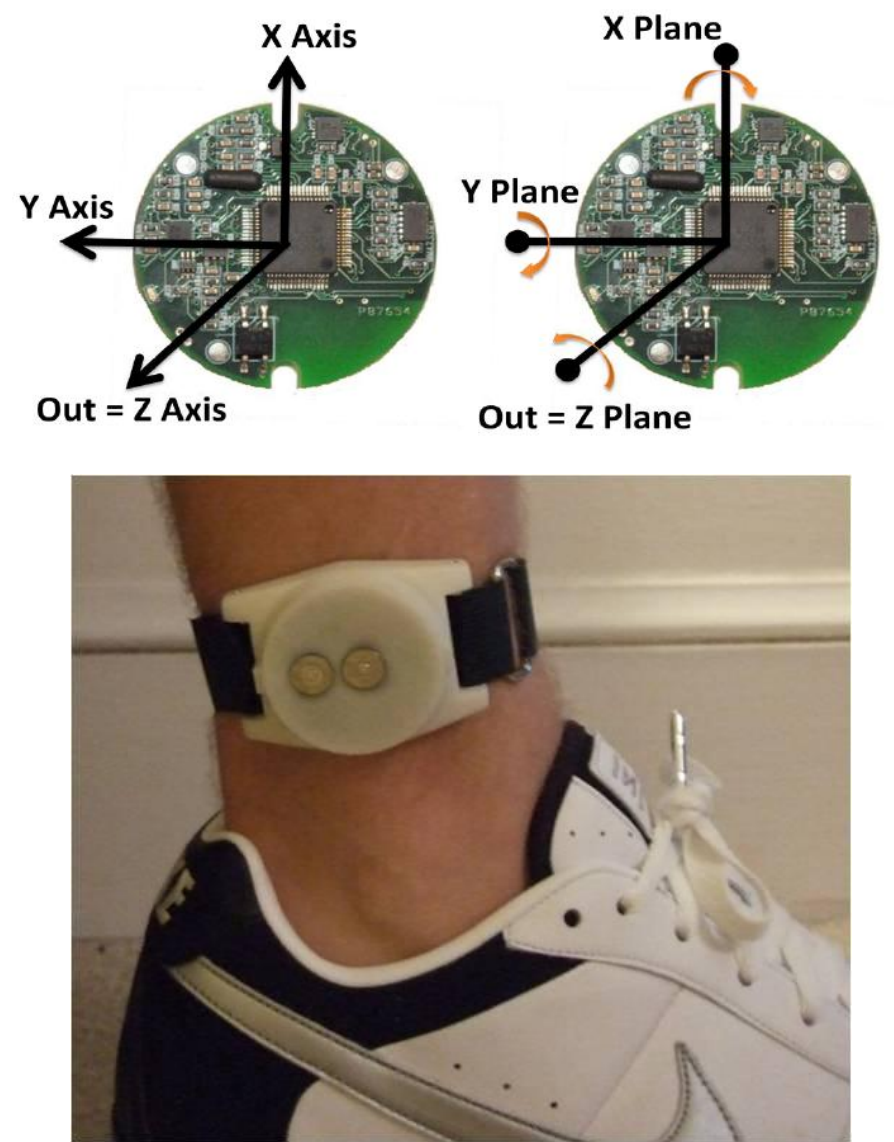

Figure 4 TEMPO Platform

At the center of the node lies the low power MSP430 microcontroller developed by Texas Instruments. By having the MSP430 on the node, we have the flexibility to perform computation on the node such as compression and classification as well as having detailed control over the node. The diagram of this system is shown in Figure 5. 


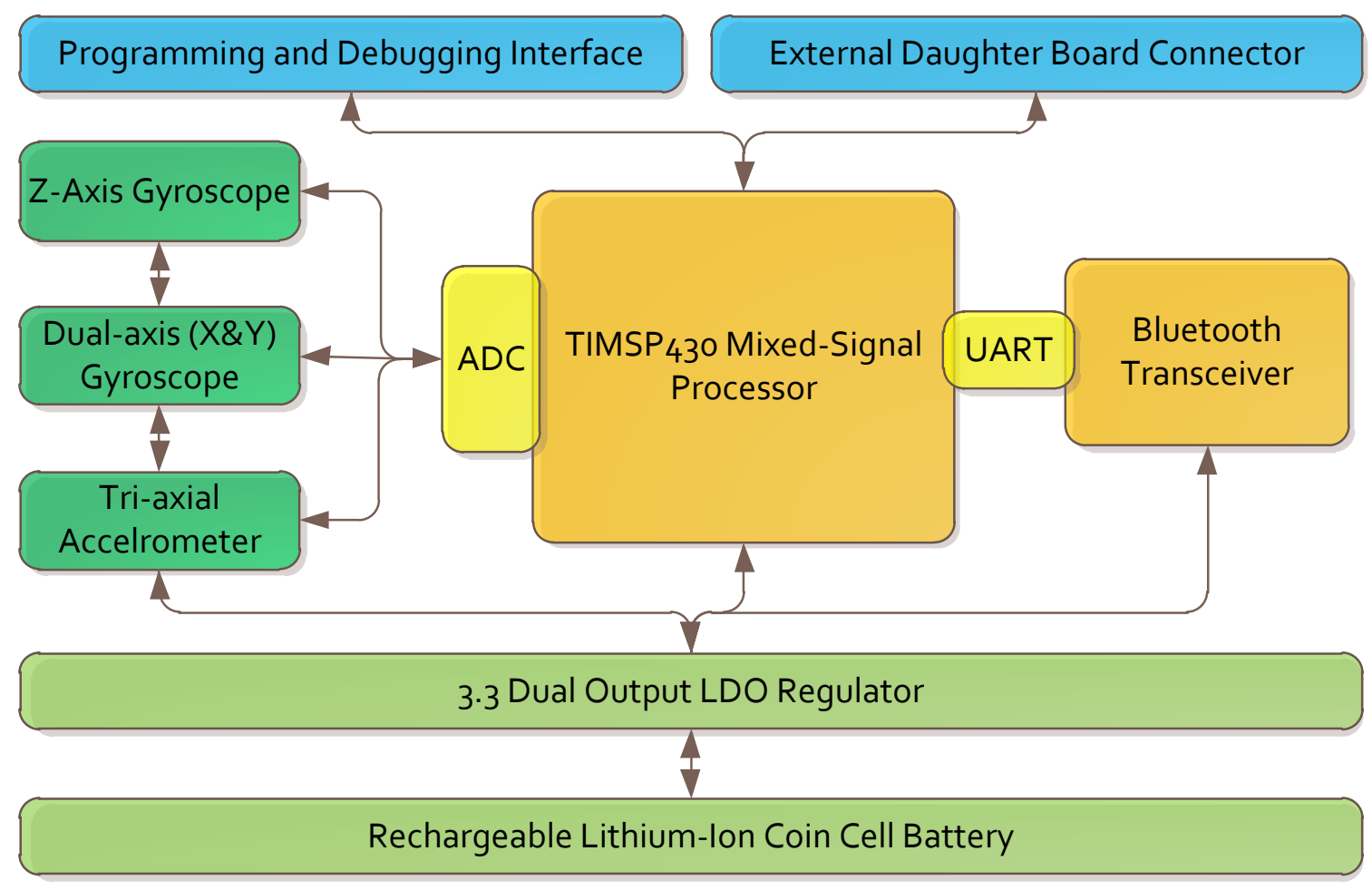

Figure 5 TEMPO Function Diagram [25]

A custom case for the TEMPO node was made in order to maintain a certain level of durability. The case contains no buttons for the user to press, but all commands are sent over a wireless channel. The case also contains a rechargeable coin cell battery and has two metal contacts that are used for recharging the device as well as turning the node on. There is also a variety of straps that can be used with the custom case that allows the node to be worn in many different locations such as the wrist, ankle, thigh, back, and anywhere else on the body. 


\subsection{Sensor Calibration ${ }^{1}$}

In order to get an accurate sensor output, sensor calibration must be performed. Accelerometers used in inertial BSN platforms are typically assumed to follow a linear model [26]. The relationship between the sensed quantity $(M)$ and the output voltage $(V)$ is described by Equation (1), which contains two key parameters: the sensitivity $(S)$ and the offset $(O)$.

$$
M=\frac{(V-O)}{S}
$$

$S$ is the ratio of voltage change to change in the physical quantity, and $O$ is the output voltage when no motion (or gravitational field, in the case of accelerometers) is applied to the sensor. These parameter values can be found in the sensor datasheets, but in practice they will deviate from the expected value.

The sources of sensor error include manufacturing variations and environmental conditions, such as the temperature, meaning that the sensitivity and offset will vary across different sensors at a given time, or within a single sensor at various times. Additionally, datasheets and white papers from manufacturers list other possible error sources, such as nonlinearity, non-orthogonality between axes, and cross-axis sensitivity. Finally, variations in chip mounting on a PCB or mounting of the PCB in the node packaging can place the sensing axes slightly out of the assumed frame of reference.

\footnotetext{
${ }^{1}$ This section was previously published in "Characterizing and Minimizing Synchronization and Calibration Errors in Inertial Body Sensor Networks,” by S. Chen, J.S. Brantley, T. Kim, J. Lach in International Conference on Body Area Networks, 2010
} 
It is impractical to individually compensate for these sources of error, and so some form of calibration is employed to holistically minimize their effect. The sensor response is checked against a known reference point (such as the gravitational field or a turntable) in order to calculate the actual sensitivity and offset by linear mapping or Newton's method $[27][28][29][30]$.

\subsubsection{Linear mapping technique}

The simplest calibration technique is the linear mapping of two points measured with respect to gravity into the linear function of Equation (1). The sensitivity $(S)$ and offset $(O)$ are calculated from these reference points according to Equation (2).

$$
S=\frac{V_{\max }-V_{\min }}{2 g}, O=\frac{V_{\max }+V_{\min }}{2}
$$

For accelerometers, each sensor axis is exposed to $1 \mathrm{~g}$ and $-1 \mathrm{~g}$ by placing it parallel with gravity.

\subsubsection{Mathematical estimation technique}

The mathematical estimation technique is based on the principle that the sum of vector magnitude of 3-axes' accelerations is $1 \mathrm{~g}$ when the accelerometer is stationary. This technique only applies for accelerometers. This principle is described by Equation (3)

$$
\sqrt{A_{x}^{2}+A_{y}^{2}+A_{z}^{2}}=1 g
$$

The three-axis accelerometer sensor is subjected to the gravitational field in six arbitrary orientations (rather than with each axis subsequently placed parallel with gravity). Each 
set of voltage measurements, $V_{x}, V_{y}, V_{z}$, gives rise to an equation of the form given by Equation (2), where $S_{i}$ and $O_{i}$ are the sensitivity and offset, respectively, of axis $i$.

$$
\begin{gathered}
F\left(S_{x}, S_{y}, S_{z}, O_{x}, O_{y}, O_{z}\right)= \\
\sqrt{\left(\frac{V_{x}-O_{x}}{S_{x}}\right)^{2}+\left(\frac{V_{y}-O_{y}}{S_{y}}\right)^{2}+\left(\frac{V_{z}-O_{z}}{S_{z}}\right)^{2}}-1=0
\end{gathered}
$$

The resulting system of six equations can be solved for the sensitivities and offsets by a mathematical iterative estimation, such as the Newton-Raphson method. An arguable advantage of this technique is that it does not require a perfectly level surface for calibration, but it does require an initial computational complexity due to the iterative estimation [28].

\subsubsection{Piecewise mapping technique}

The previous techniques assume a linear sensor model, but a piecewise linear equation may better fit the nonlinearities in the sensor response. This procedure requires profiling the sensor against more than two known reference points and mapping the readings into a piecewise linear function. For an n-point linear function, the $n^{\text {th }}$ sensitivity and offset are given as follows:

$$
S(n)=\frac{\left(V_{n}-V_{n-1}\right)}{\left(\cos \theta_{n}-\cos \theta_{n-1}\right) g}, O=\frac{V_{n}+V_{1}}{2}
$$

with $n$ being the number of required measurement points. The most convenient and commonly-used choice of $n$ is 3 data points, such as $0^{\circ}, 90^{\circ}$, and $180^{\circ}$. However, increasing the number of data points increases the conversion accuracy, as detailed in the Chapter 5 . 


\section{Chapter 4 Forward Head Posture Detection}

Accelerometers have long been used in many industries (airplane, car etc.) to detect inclination. An accelerometer mounted on a human neck and back can be used to detect FHP by assessing the CV angle. It can also provide continuous monitoring and real-time bio-feedback to prevent neck pain caused by FHP. The TEMPO system is used as the platform to validate this concept. In this Chapter, the $\mathrm{CV}$ angle detection algorithm will be introduced. The design of FHP monitoring and bio-feedback system will be described as well. 


\subsection{Number and Location of Sensors}

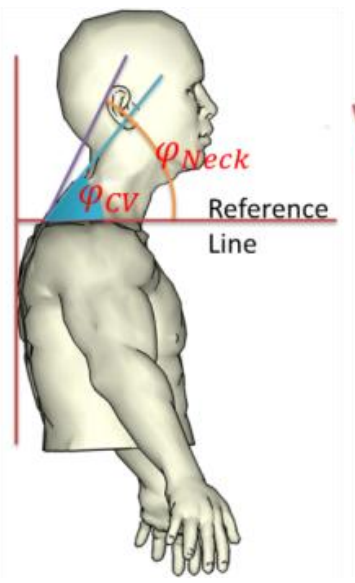

(a)

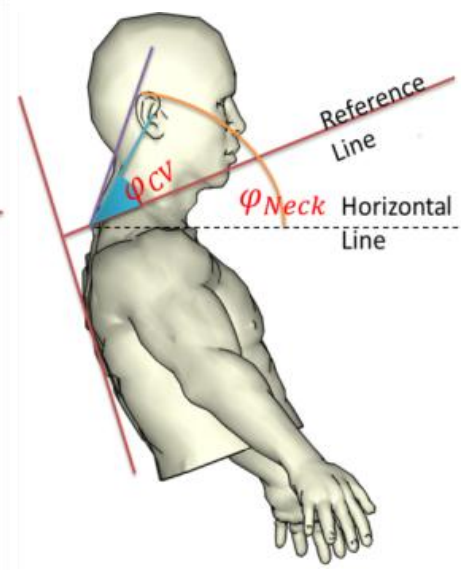

(b)

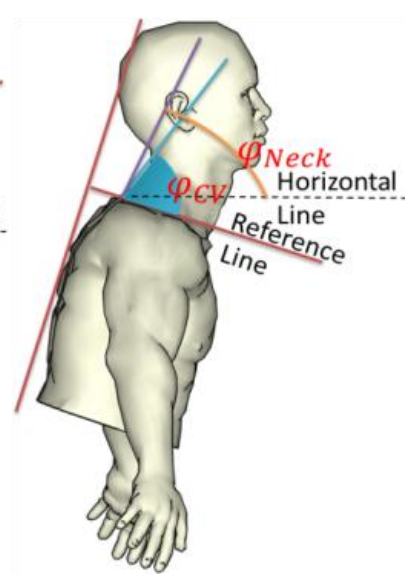

(c)

Figure 6 Need for Multiple Sensors

Figure 6 shows the consideration of the requirement of multiple sensors for the mobility of detection. Three common postures are carefully considered. In Figure 6 (a), the subject is standing or sitting with his torso upright and head forward. This is the posture posed in most clinical assessment situations. This posture is commonly observed in people who are exposed to bad ergonomics or have bad walking posture. In Figure 6 (b), the subject is leaning his torso backwards while extending his head forward. This is commonly observed in sitting positions, such as when a person is sitting on a couch watching TV or leaning against a chair while reading from a computer. Although the neck angle is large with respect to the horizontal line, the spine actually bears a heavy load because the torso is leaning backwards. In Figure 6 (c), the subject is leaning his torso forward, with the neck angle indicating a small angle with respect to the horizontal line. However, the subject's spine is not bearing much weight since his torso is leaning 
forward as well. This implies that assessing the neck angle alone FHP will miss detection of FHP as shown in Figure 6 (b). Additionally, the system may register false alarm in the scenario shown in Figure 6 (c). Thus, measuring with multiple sensors is more reasonable to detect $\mathrm{CV}$ angle because the reference line is changed for various postures.

In order to validate this concept, a preliminary test was conducted using three sensor location combinations - head, neck, and back as shown in Figure 7 - and a correlation analysis was performed on the information provided by each sensor. As seen in Table 1, there is a strong correlation coefficient between the neck angle and head angle among the 4 subjects ( 3 health subjects and 1 with FHP), which implies that the neck angle and head angle change in the same direction with same magnitude. With this result, just two sensors - neck and back - were used to measure CV angle.

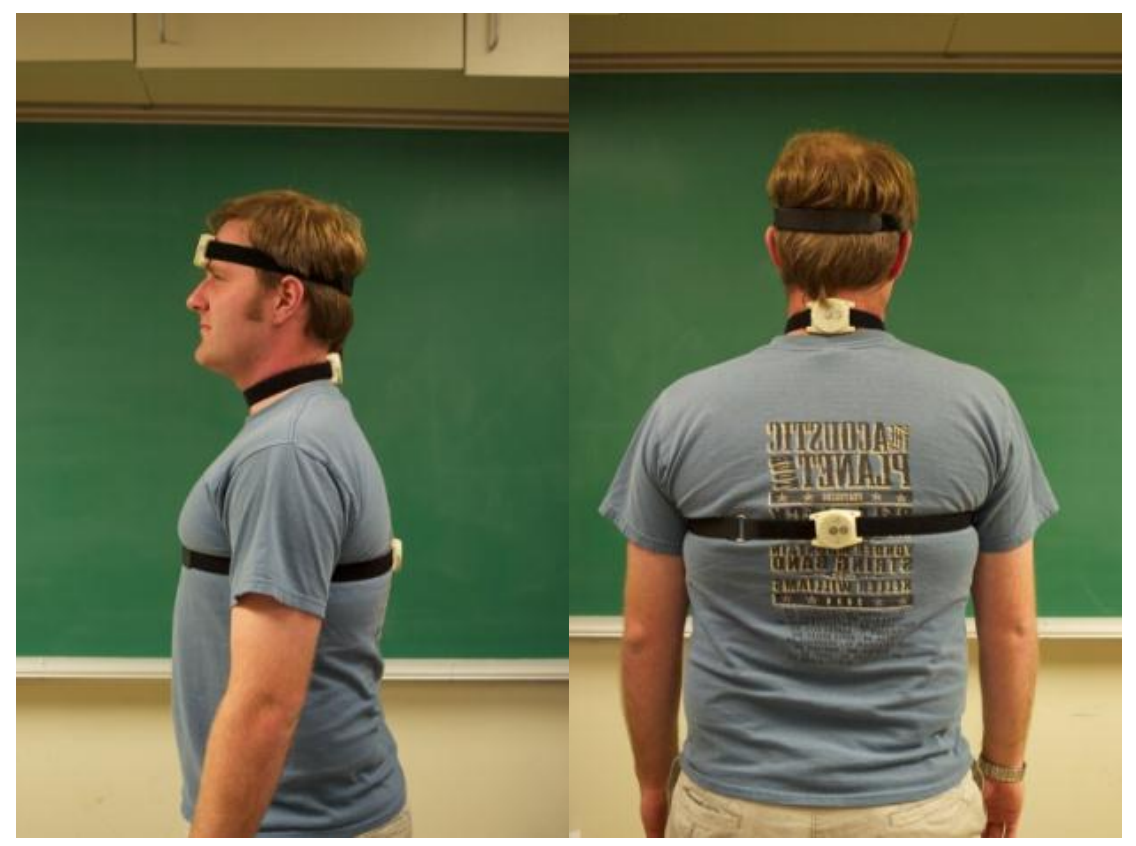

Figure 7 Sensor Location Experiment 
Table 1 Correlation Coefficient of Nodes Position

\begin{tabular}{|c|c|c|c|c|}
\hline \multirow{2}{*}{$\begin{array}{c}\text { Subject } \\
\text { Type }\end{array}$} & \multirow{2}{*}{ Subject ID } & \multicolumn{3}{|c|}{ Correlation Coefficients } \\
\cline { 3 - 5 } & $\begin{array}{c}\text { Neck- } \\
\text { Head }\end{array}$ & Neck-Back & Head-Back \\
\hline \multirow{2}{*}{ Healthy } & Subject 2 & 0.9555 & 0.8627 & 0.6841 \\
\cline { 3 - 5 } & Subject 1 & 0.9640 & 0.7517 & 0.7736 \\
\cline { 3 - 6 } & Subject 3 & 0.9390 & 0.8181 & 0.6154 \\
\hline \multirow{2}{*}{ FHP } & Subject 4 & 0.9048 & 0.6049 & 0.3254 \\
\hline
\end{tabular}

The next step is to understand the node position on the selected locations. In order to know the right position of sensor on the neck, 2 subjects (1 healthy and 1 FHP) were measured for each mounting on the neck as seen in Table 2. It shows the angle error between EHPI and TEMPO. When the sensor is mounted on different positions of the neck, the angle errors vary. The smallest angle error was observed at 6 degrees on the back of the neck. Therefore, it shows that the best place to mount the sensor is on the back of the neck. In addition to the neck position, the back position differences were not significantly changed. 


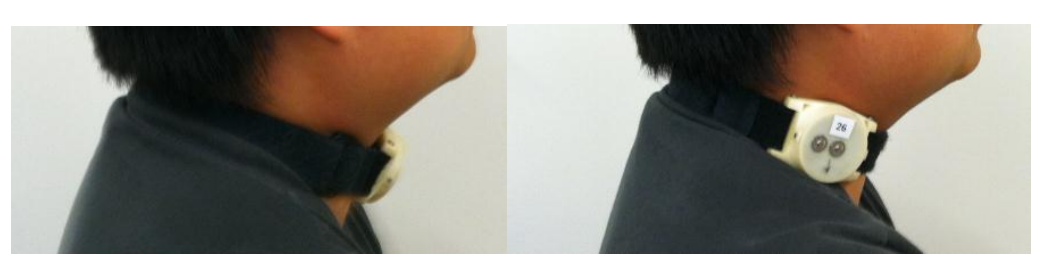

(a) Forth

(b) Side

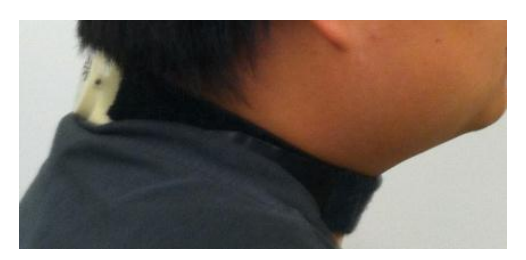

(c) Back

Figure 8 Node Position on the Neck

Table 2 The Measurement for Node Position on the Neck

\begin{tabular}{|c|c|c|c|c|}
\hline \multirow{4}{*}{ Subject } & $\begin{array}{c}\text { Neck Mounting } \\
\text { Position }\end{array}$ & EHPI & TEMPO & $\begin{array}{c}\text { Angle Error } \\
\text { (deg) }\end{array}$ \\
\hline \multirow{3}{*}{ Healthy } & Back & 72.4 & $64.6 \pm 0.4$ & 7.8 \\
\cline { 2 - 5 } & Forth & 72.4 & $59.2 \pm 0.4$ & 13.2 \\
\cline { 2 - 5 } & Side & 72.4 & $46.2 \pm 0.5$ & 26.2 \\
\hline \multirow{5}{*}{ FHP } & Back & 58.6 & $52.6 \pm 0.4$ & 6 \\
\cline { 2 - 5 } & Forth & 58.6 & $52.4 \pm 0.4$ & 6.2 \\
\cline { 2 - 5 } & Side & 58.6 & $44.8 \pm 0.4$ & 13.8 \\
\hline
\end{tabular}




\subsection{Measurement}

Figure 9 shows how the CV angle can be measured using body-mounted accelerometers, such as the wireless inertial BSN system TEMPO 3.1 [24] used in this work. The segment angles are computed as shown in Equations (6)-(8).

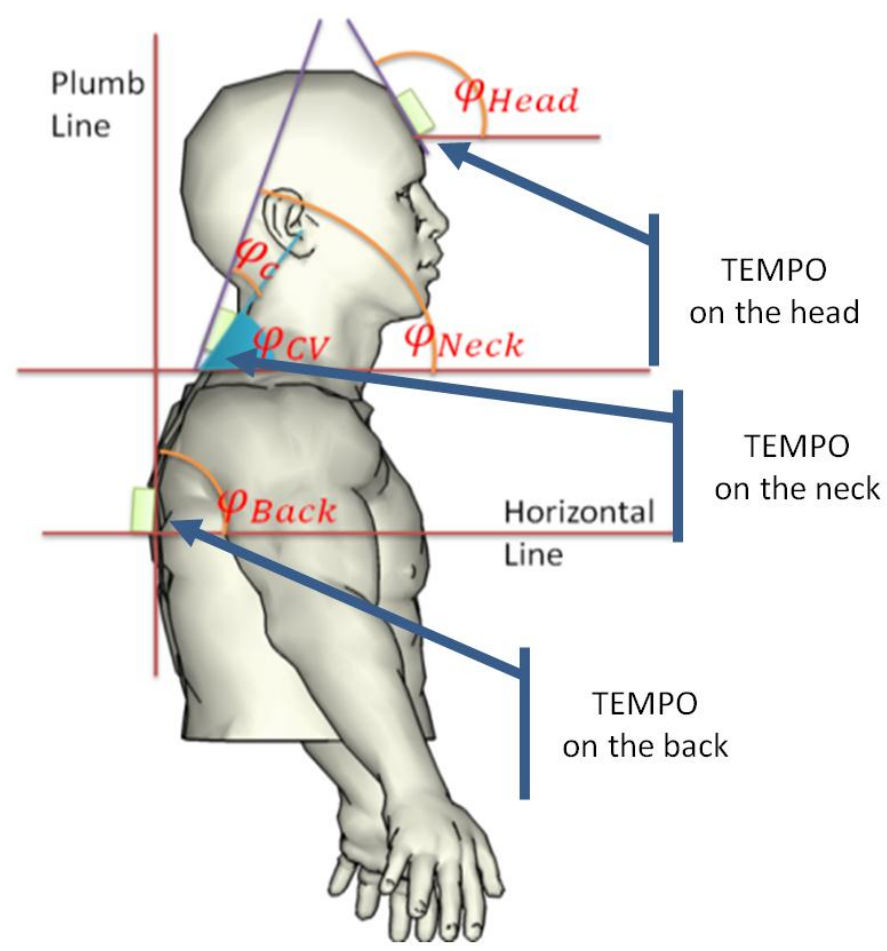

Figure 9 CV Angle

$$
\begin{gathered}
\varphi_{\text {neck }}=\arctan \left(\frac{A_{z}}{\sqrt{A_{x}^{2}+A_{y}^{2}}}\right) \\
\varphi_{\text {head }}=\arctan \left(\frac{A_{z}}{\sqrt{A_{x}^{2}+A_{y}^{2}}}\right)
\end{gathered}
$$




$$
\begin{aligned}
& \varphi_{\text {back }}=\arctan \left(\frac{A_{z}}{\sqrt{A_{x}^{2}+A_{y}^{2}}}\right) \\
& \varphi_{c}=\varphi_{\text {Neck_InitialPosture }}-\varphi_{E H P I} \\
& \varphi_{B}=\varphi_{\text {Back_InitialPosture }}-90
\end{aligned}
$$

where $\varphi_{\text {head }}, \varphi_{\text {neck }}$ and $\varphi_{\text {back }}$ indicates the inclination of the respective segments with respect to a horizontal line. Since different individual body contours cause an offset between the neck angle and the $\mathrm{CV}$ angle, and the back angle and the reference line, the offsets $\varphi_{c}$ (the difference between the EHPI system [18] measured CV angle and the accelerometer measured neck angle) and $\varphi_{B}$ (the difference between 90 degrees and the accelerometer measured back angle) should be found in the initial calibration, during which the subject is positioned upright.

As discussed in the explanation following Figure 7, the back angle must also be considered in the $\mathrm{CV}$ angle calculation. Moreover, because of the high correlation coefficients of the neck and head, the neck and back angle measurement were reasonable points to measure $\mathrm{CV}$ angle. Therefore, the $\mathrm{CV}$ angle equation may be found by the neck and back as below in Equation (11).

$$
\varphi_{C V}=\left(\varphi_{N e c k}-\varphi_{C}\right)+90-\left(\varphi_{\text {Back }}-\varphi_{B}\right)
$$

where $\varphi_{N e c k}$ and $\varphi_{B a c k}$ are computed in (6) and (8). 


\subsection{Real-time Monitoring System}

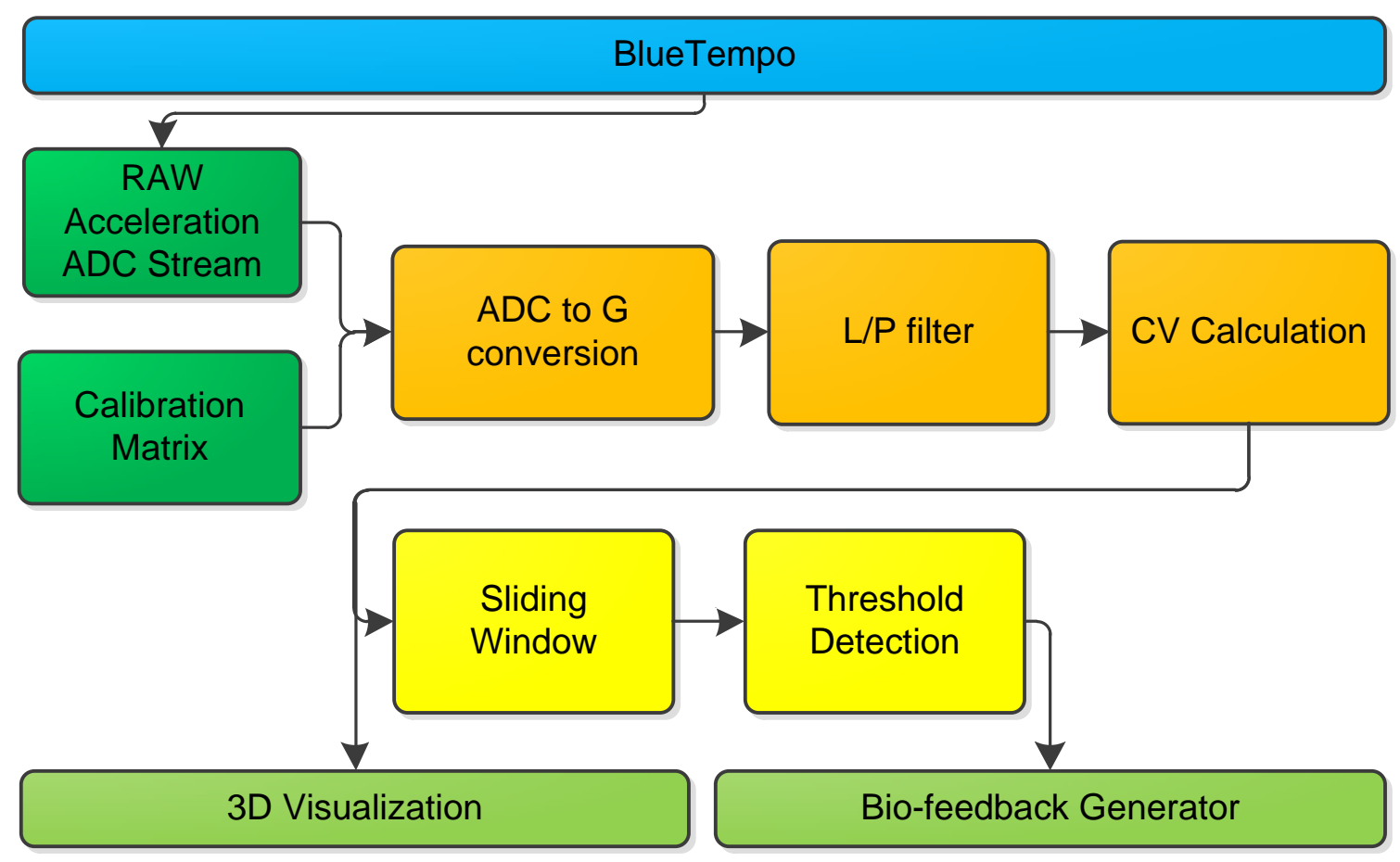

Figure 10 FHP Monitoring System Functions

A LabView ${ }^{\circledR}$ program was developed to continuously monitor the $\mathrm{CV}$ angle and generate bio-feedback. Figure 10 shows the functional block diagram of a real-time FHP monitoring system. Five major functions are implemented for this system in Figure 10. $\mathrm{ADC}$ to $\mathrm{G}$ conversion is to transform the ADC output of TEMPO to a gravity value with regard to the real angle of the TEMPO as mentioned in Equation (1). A low-pass filter was applied to reduce small noise. The CV calculation uses Equation (11) to calculate the $\mathrm{CV}$ angle using the angles of the neck and back. The sliding window block is to extract standard deviation from the given sliding windows of streaming data to not provide biofeedback during non-static periods, as the calculated angles are unlikely to be correct and 
could result in overly frequent false alarms. A 5 second sliding window and 2 degrees standard deviation threshold were chosen by simple experiment. To get reasonable values, the simple angle measurement was conducted, while the subject was the stable without any movement. However, those values should be optimized in the future through additional studies. 45 degree of CV angle [18] was used to detection FHP in the threshold block. When the detection algorithm suspects that a subject is engaging in a FHP posture, the software generates an output identifying the subjects with a severely decreased CV angle. Below is the overall algorithm for FHP detection and bio-feedback:

\section{FHP Detection Algorithm}

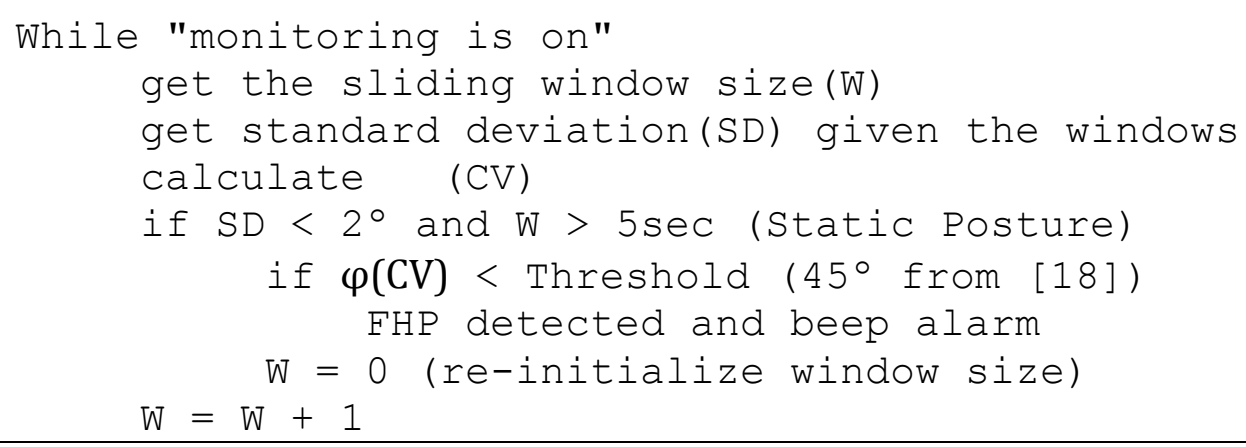




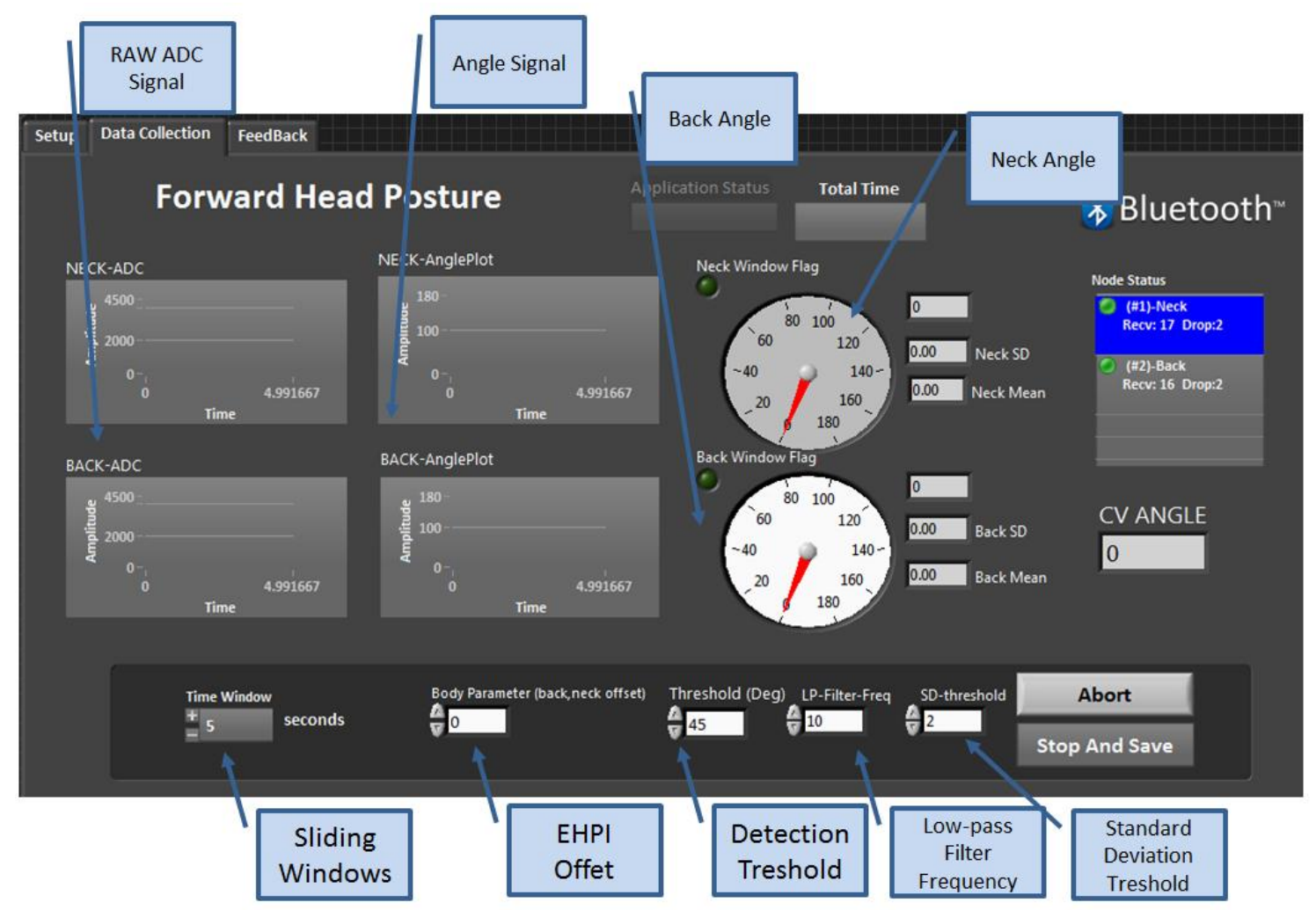

\section{Figure 11 FHP Monitoring Interface}

On this interface in Figure 11, two angle measurements are displayed. Before using this system, the subject is required to mount the TEMPO node on the neck and back, while standing behind the EHPI system to get the initial individual parameters, i.e. the initial neck angle and back angle as stated in Equation (11). Next, the offset is computed and inputted as Body Parameter in the interface as shown in Figure 11. The threshold is also input into the interface. Given the issues of various body shapes and variable node mounting, the patients need to measure the initial offset in clinic at least once for continuous monitoring. 


\subsection{Bio-feedback}

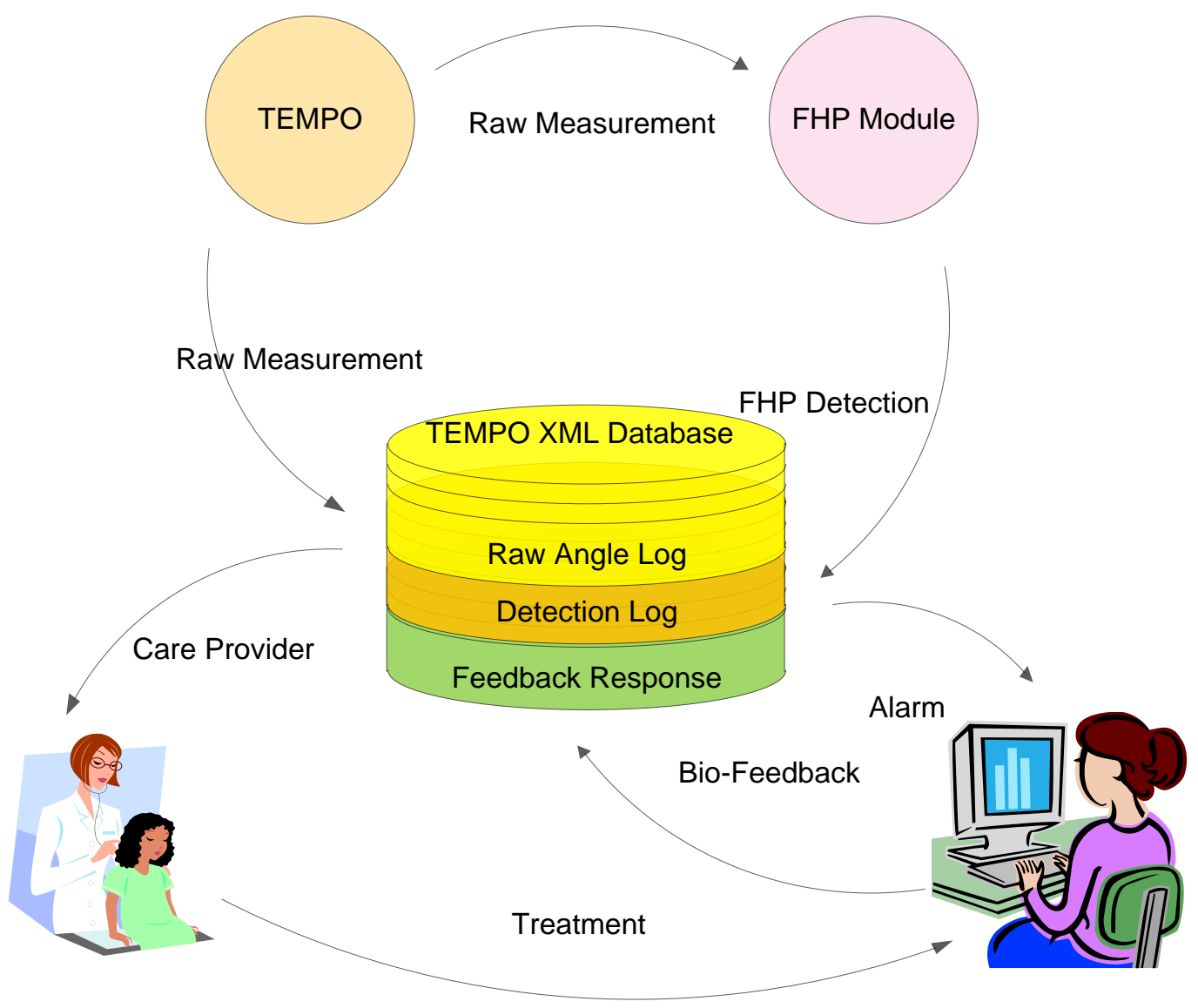

Figure 12 Bio-Feedback Flow Diagram 


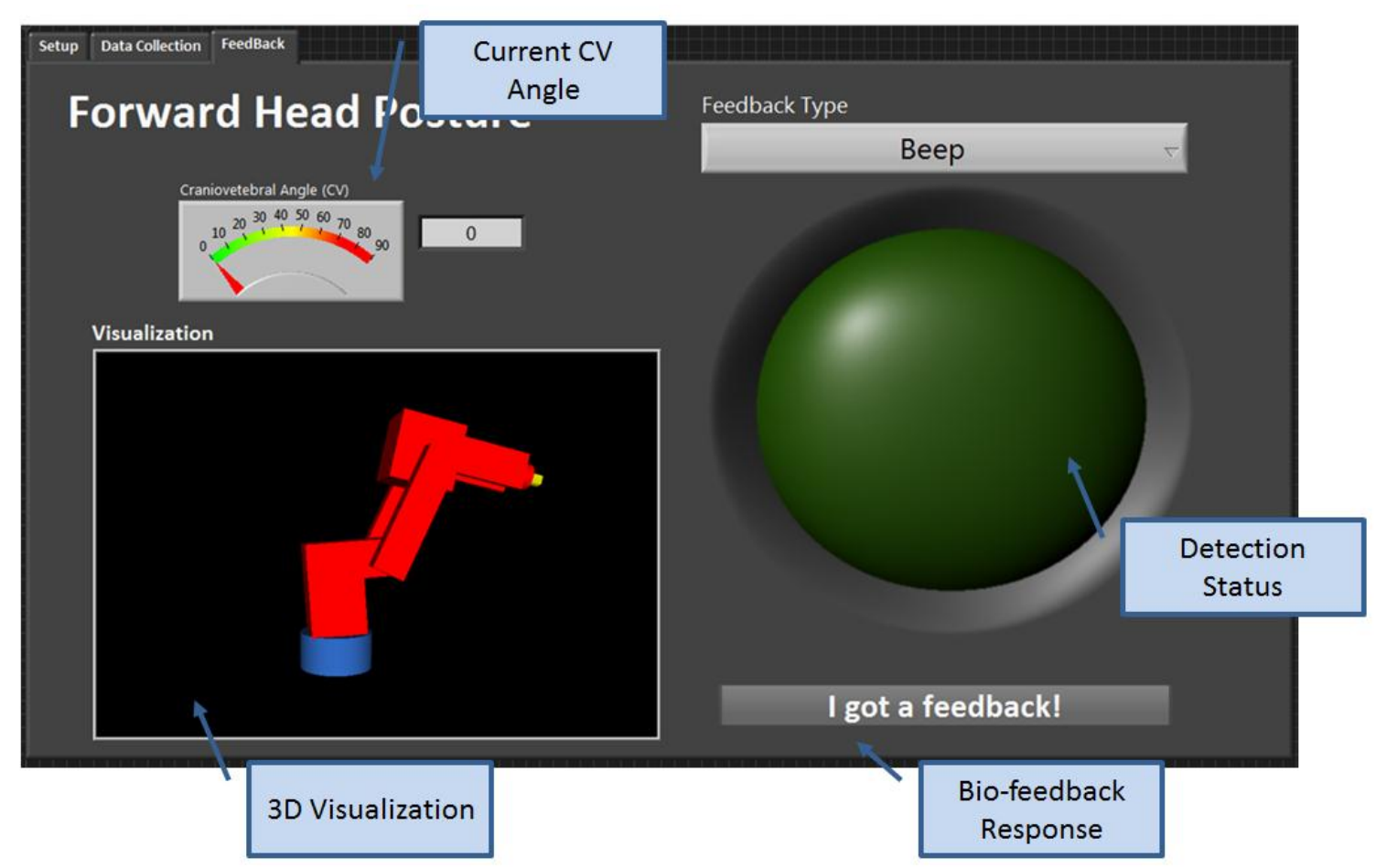

Figure 13 FHP Feedback Display

Figure 12 shows the mechanism of bio-feedback for FHP. With the TEMPO platform, the FHP monitoring beeps when the program detects FHP. A data log collects raw data and saves the detection status and generates feedback information for subsequent analysis as shown below in Chapter 5. Bio-feedback alerts the user of improper posture so the user can self-correct his posture. Once the user is alerted, the user can provide feedback to the system to indicate whether or not the user heard the alarm. This feature is implemented to analyze the effectiveness of the bio-feedback function. In addition, FHP feedback display showed 3D real-time of the user's body so he can adjust his posture accordingly. 


\section{Chapter 5 Experimental Results}

In this chapter, the detection accuracy and the effectiveness of the bio-feedback function are explored. With respect to the detection accuracy, the angle comparisons of the various postures were compared to test the performance. To measure the effectiveness of the biofeedback feature, a comparison was made between how much time subjects spent in FHP with and without bio-feedback.

\subsection{Sensor Calibration Result}

In order to accurately test and measure the angles obtained by the accelerometer, a digital inclinometer instrument with $\pm 0.5^{\circ}$ error was used. Figure 13 shows the experimental setup of the accelerometer for calibration and measurement. The TEMPO node is placed in a custom bracket used to precisely place it in the desired frame of reference.

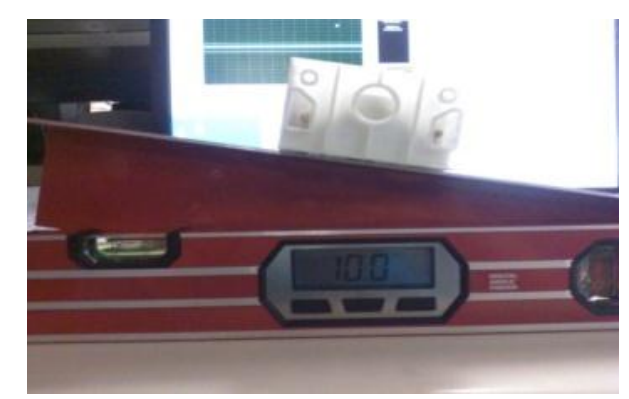

Figure 14 Calibration and Measurement Instrument 
Table 3 shows a comparison among 4 calibration methods for the accelerometer, including piecewise mapping with $\mathrm{n}=3,5$, and 7 . Datasheet conversion refers to the baseline case using the sensitivity and the offset listed in the sensor datasheet. In order to verify the output, measurements were taken at $10^{\circ}$ increments from $0^{\circ}$ to $180^{\circ}$ on the inclinometer. Piecewise calibration resulted in the lowest RMSE, with more data points providing higher accuracy, although the additional efforts to obtain a high $\mathrm{n}$ value must be balanced against the accuracy improvements. The piecewise $(n=7)$ must have 7 fixed and precise data points, but the Newton Raphson method required 6 arbitrary data points. Therefore, we chose Newton Raphson method for this system since the error difference is less than 1 degree and the calibration process is simpler.

Table 3 Accuracy Analysis of 1-axis Calibrated Accelerometer

\begin{tabular}{|c|c|c|}
\hline Calibration Techniques & RMSE & $\begin{array}{c}\text { Mean of } \\
\text { Angle Error (deg) }\end{array}$ \\
\hline Datasheet Conversion & 22.75 & 20.95 \\
\hline Newton & 1.63 & 0.26 \\
\hline Linear & 1.53 & 0.24 \\
\hline Piecewise (n=3) & 1.51 & 0.22 \\
\hline Piecewise $(\mathbf{n}=5)$ & 0.78 & 0.20 \\
\hline Piecewise $(\mathbf{n}=7)$ & 0.70 & 0.15 \\
\hline
\end{tabular}




\subsection{Validation System Setup}

To verify the CV detection algorithm, an EHPI [18] system was used to assess the initial CV angle. In Figure 15, an inclinometer was mounted on a tripod with an omnidirectional gimbal, which was used to adjust the inclinometer. During the assessment, the subject stood upright behind the EHPI system. The experimenter observed the CV angle in front of the EHPI system. The observer checked the level meter on the inclinometer to make sure the device was on a leveled surface. The inclinometer reading was the $\mathrm{CV}$ angle measured by this EHPI instrument setup. This angle was also used to calibrate the initial $\mathrm{CV}$ angle measured by the TEMPO system as mentioned in Chapter 4.1. The validation system is captured as seen in Figure 16.

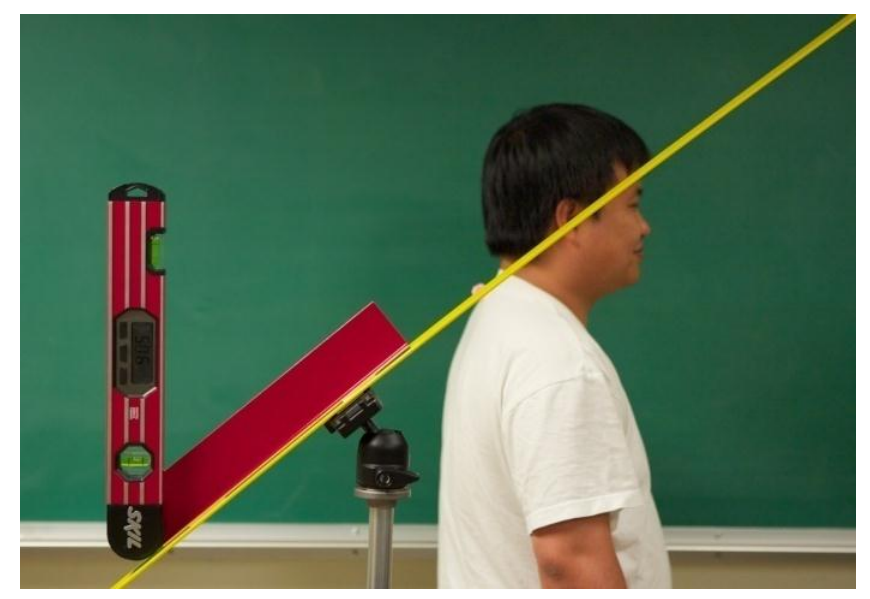

\section{Figure 15 Validation System Setup with EHPI for Initial Posture}

Considering that this method is subject to flexibility of the subject and space limit during the measurement, I took the common image processing application called the Tracker [31] to verify my method. However, I relied on the EHPI system to measure the initial CV angle in order to obtain better accuracy for the $\mathrm{CV}$ angle measurement of the standing 
position. I took the approach as described in [22]. I recorded videos of the subject's profile while the TEMPO system measured the CV angle. Then the angles on the video were calculated by the Tracker [31].

However, the validation methods suffer from a parallax problem when a human observer is reading the inclinometer or taking pictures. Ideally, a 3D motion capture system such as Vicon ${ }^{\circledR}$ should be used to obtain better accuracy. But for the purpose of this pilot study, I adopted the validation method used in previous research.

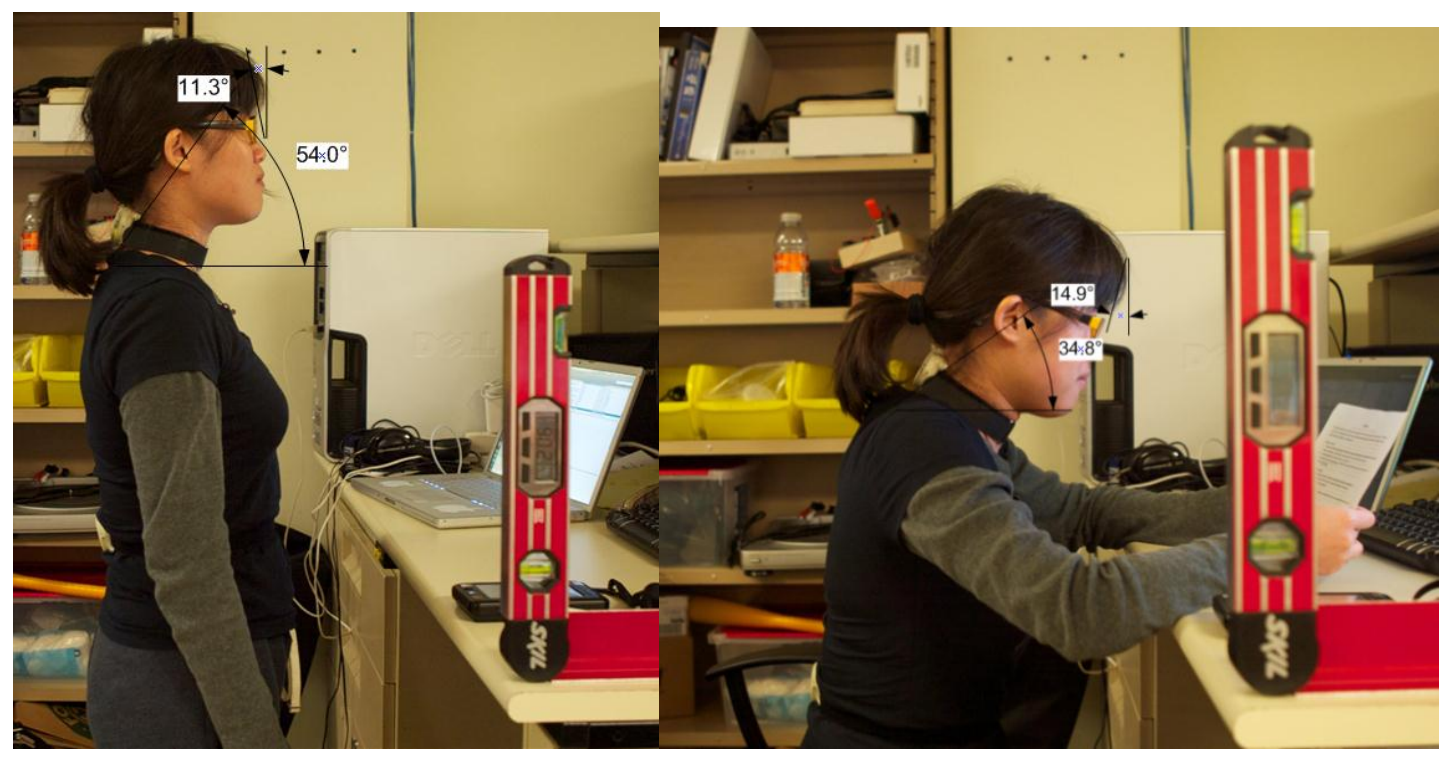

Figure 16 Validation with Image Processing for Various Postures

\subsection{Detection Accuracy}

In order to see detection accuracy, two subjects, one with FHP history and one without, were recruited for this experiment. Two TEMPO nodes were securely but comfortably mounted on the neck and back with elastic Velcro® straps. Both subjects simulated their daily activities, while TEMPO data was continuously streamed to the LabView® 
program. The video was inspected using an image processing application to assess FHP and evaluate the accuracy of the BSN-based FHP detection algorithm.

Table 4 CV Measurement for Various Postures

\begin{tabular}{|c|c|c|c|}
\hline & Measured (degree) & Validation (degree) & $\begin{array}{c}\text { Angle Error } \\
\text { (degree) }\end{array}$ \\
\hline Posture 1 & 54.7 & 54 & 0.7 \\
\hline Posture 2 & 68.9 & 45 & 23.9 \\
\hline Posture 3 & 48.5 & 34.8 & 13.7 \\
\hline Posture 4 & 64.5 & 47.1 & 17.4 \\
\hline Posture 5 & 66.3 & 46.1 & 20.2 \\
\hline Posture 6 & 36.6 & 52.7 & 16 \\
\hline Posture 7 & 11.2 & 47.1 & 13 \\
\hline Posture 8 & 42.8 & 65 & 22 \\
\hline Posture 9 & 19.9 & 33 & 13.1 \\
\hline Posture 10 & 52.9 & 48.8 & 13 \\
\hline Posture 11 & 15.5 & & 4.1 \\
\hline
\end{tabular}


The 11 different positions are listed below:

1. Initial posture (Standing upright)

2. Reading from a computer monitor (while sitting on a couch)

3. Reading a newspaper/book by a desk/table

4. Sitting on a stool (without doing anything)

5. Sitting on a chair (without doing anything)

6. Relaxing in on a chair (laid back posture)

7. Feet on the desk with laptop on the lap (head lower than the top of the chair)

8. Crouching in on a couch (reading/watching TV)

9. Crooked back while walking/standing

10. Typing on a desktop/laptop

11. Writing on paper on a desk

Table 4 shows the comparison between the CV angles measured by the FHP monitoring system and the validation result. This result shows mobility and accuracy of the FHP monitoring system. As seen in Table 4, some of the postures have larger errors compared to the standing posture because the sensor mounting and dynamic body movements caused variations. The errors on postures $2,5,7$ and 8 were high values because they measured while the body was being placed on the soft material or pushing the sensor node. The postures in those situations had a big impact on the FHP detection, so we were using postures $1,3,4,6,9,10$ and 11 for the following overall detection test.

A sample of the results for FHP detection accuracy is shown in Table 5. One healthy subject was measured for 30 minutes in the various postures with a one second granularity (i.e., each count in Table 5 represents one second). The overall precision of 88.2\% and accuracy of $94.5 \%$, shows reasonable FHP detection efficacy, but additional research is necessary to determine what the efficacy requirements are for the ultimate application. 
Table 5 Detection Accuracy Validated by Video

\begin{tabular}{|l|c|c|c|}
\hline \multicolumn{2}{|l|}{} & \multicolumn{2}{c|}{ Video Validation } \\
\cline { 3 - 4 } & \multicolumn{2}{c|}{ FHP Flag } & No Flag \\
\hline \multirow{2}{*}{ TEMPO Solution } & FHP Flag & 195 (True positive) & 26 (False negative) \\
\cline { 2 - 4 } & No Flag & 73 (False positive) & 1511 (True negative) \\
\hline
\end{tabular}

Through this experiment, it is determined that standard deviation threshold and sliding window size affects the system performance. If the standard deviation threshold is reduced, the system may inappropriately provide bio-feedback in non-static postures, resulting in false alarms. Conversely, if the threshold is increased, the system may not provide bio-feedback when a static FHP posture is indeed present (false negatives). Similar effects are seen when varying sliding window size. Finding the appropriate values for these parameters based on tradeoffs between false positives (which can create compliance issues) and false negatives (which can reduce the overall effectiveness of the system) is part of future work and requires additional human subject testing.

\subsection{Evaluation of the Bio-feedback}

The effectiveness of the bio-feedback function was evaluated by comparing data sessions with and without bio-feedback. 4 subjects were measured for each 30 minutes. Overall, 
bio-feedback helped decrease the percentage of time the subject spent in FHP, as shown in Figure 17. Subject 2 with a history of FHP also showed a significant decrease in time spent in FHP with bio-feedback. On average, the system provided 2.9 bio-feedback beeps per minute, which may raise compliance issues during extended use (i.e. the user may ignore the beeps or shut off the system altogether) even if that feedback is based on true positives.

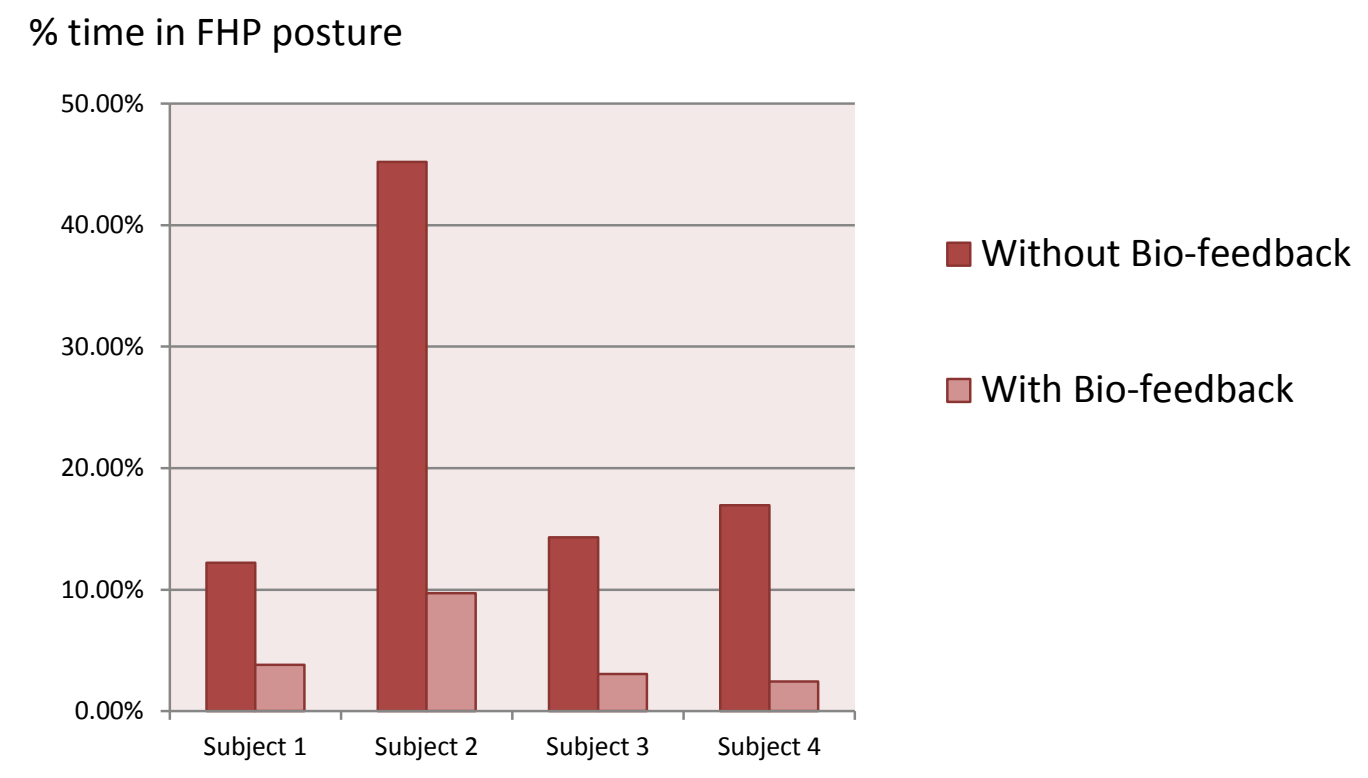

\section{Figure 17 Effectiveness of Bio-feedback with FHP Monitoring}

The result of this experiment demonstrates the importance of bio-feedback so as to induce FHP posture correction. In order to find the optimal frequency of a bio-feedback, additional research into ergonomics and psychology is necessary. In addition, further experiments should be conducted using various sliding window sizes, standard deviation thresholds, and CV angle thresholds to explore the potential tradeoffs between efficacy (i.e. FHP reduction) and compliance. Moreover, in order to provide an effective 
biofeedback, optimal frequency and different types of bio-feedbacks should be studied and tested in the future. 


\section{Chapter 6 Conclusion and Future Work}

This thesis investigated a promising solution for continuously monitoring head posture of people afflicted with FHP, a disorder that affects a large percentage of the population. Using accelerometer-based wireless inertial body sensors, a continuous monitoring method was implemented. Sensor calibration methods were explored to achieve better angle measurement. To determine correct $\mathrm{CV}$ angle measurement, of the location of the sensor on human body was explored. The detection algorithm and bio-feedback were evaluated with high accuracy.

To further realize the potential of this application, additional research is necessary to determine the most appropriate sensor solution and bio-feedback platform. Since the TEMPO is primarily designed as a wrist mount, a smaller sensing platform should be designed for the head and neck mount. In order to make the system more practical, sensor placement in everyday items like hats or glasses should be considered.

In addition, more human subject experiments need to be performed to refine and comprehensively evaluate the detection algorithms and the bio-feedback in order to maximize efficacy and compliance. Lastly, the frequency and the form of bio-feedback should be researched as the follow-up study. 


\section{Appendix 1 : FHP Design-Entire Block}

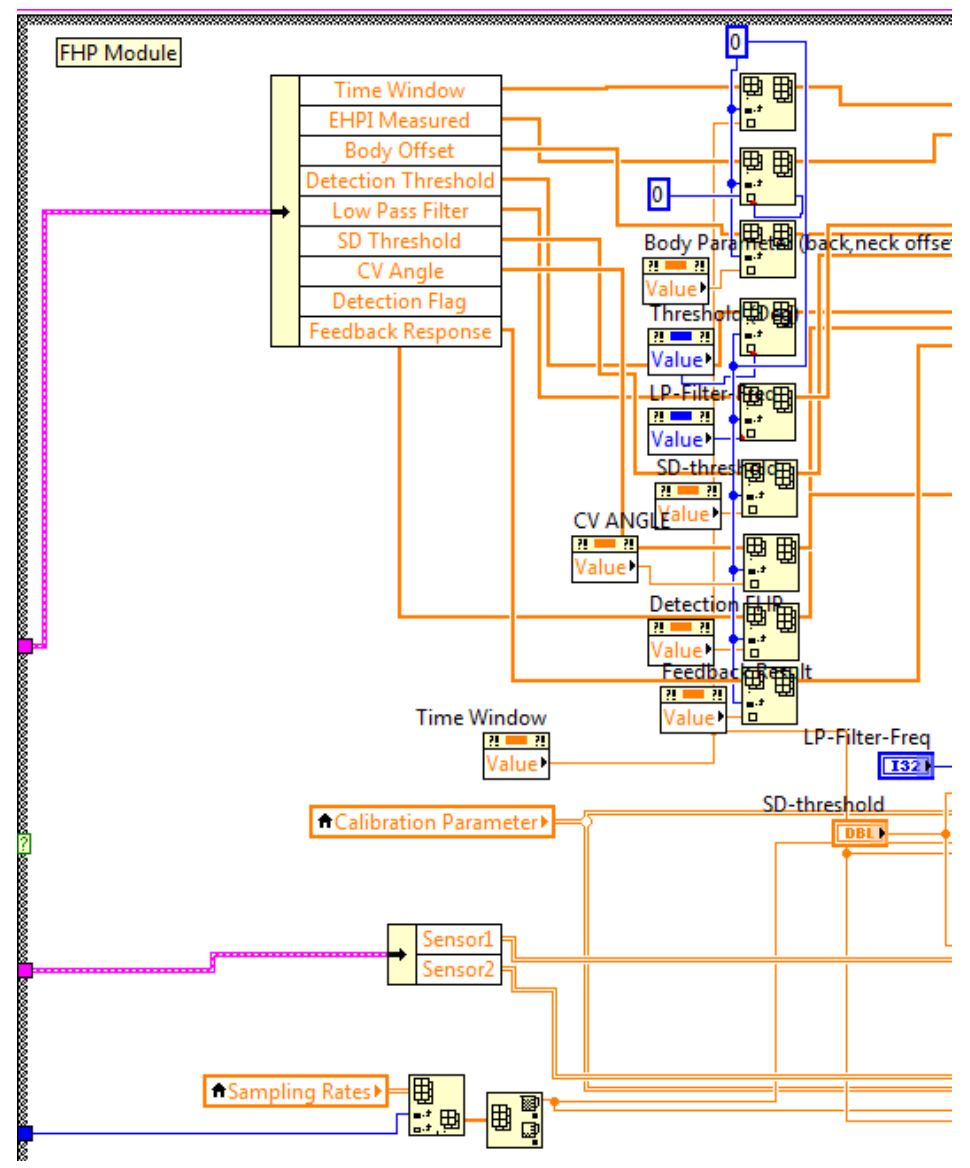




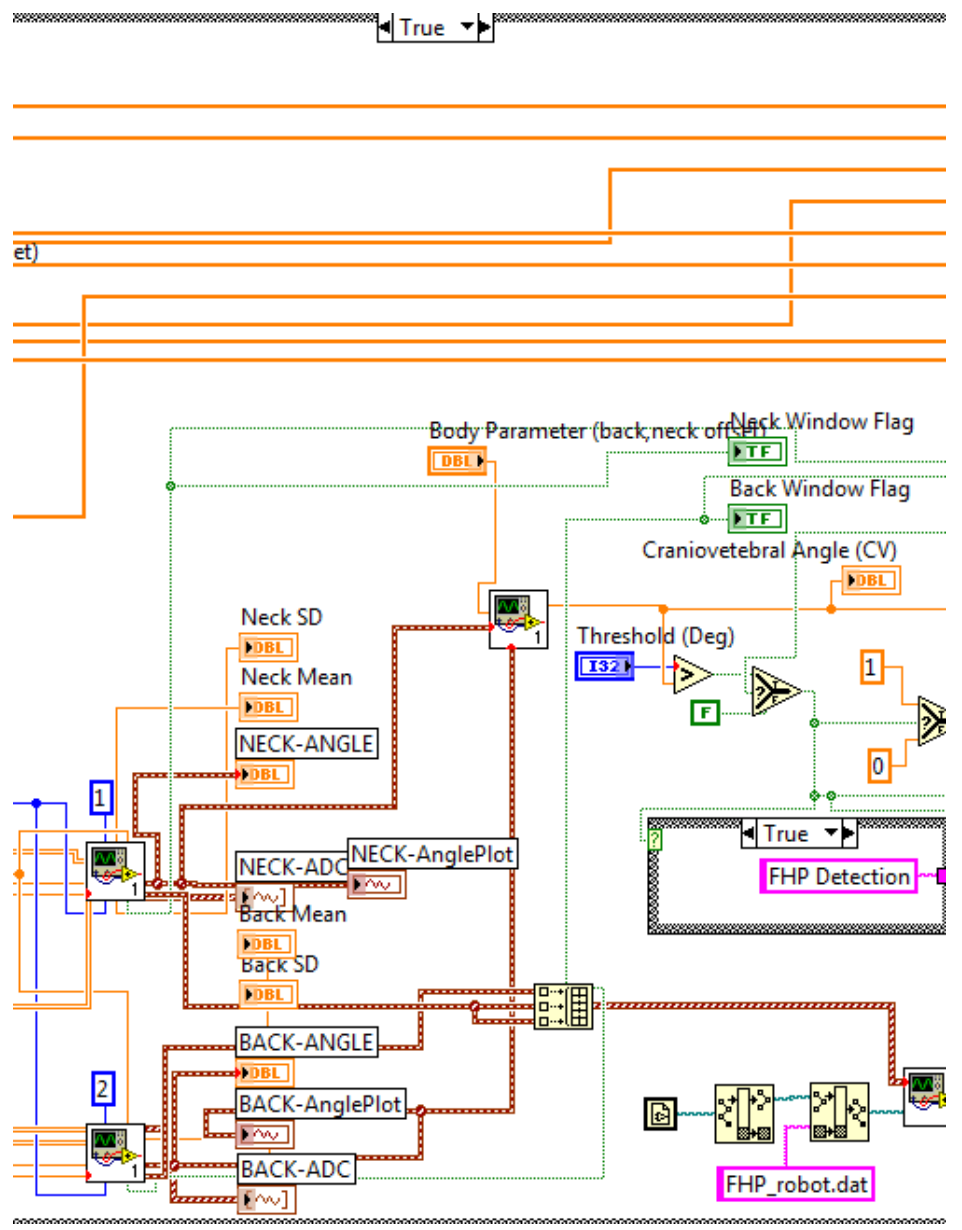




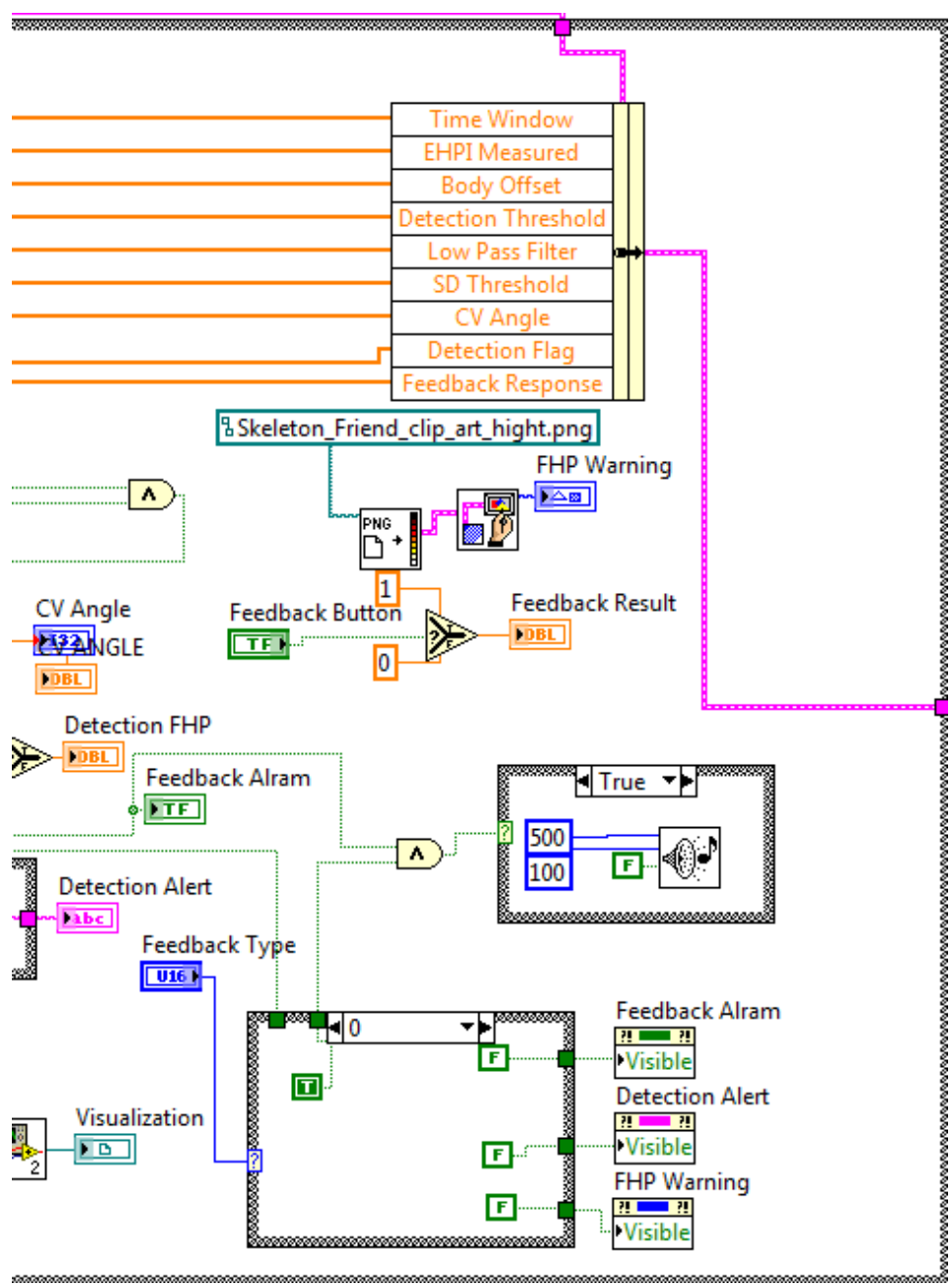




\section{Appendix 2 Conversion/Window/LP}
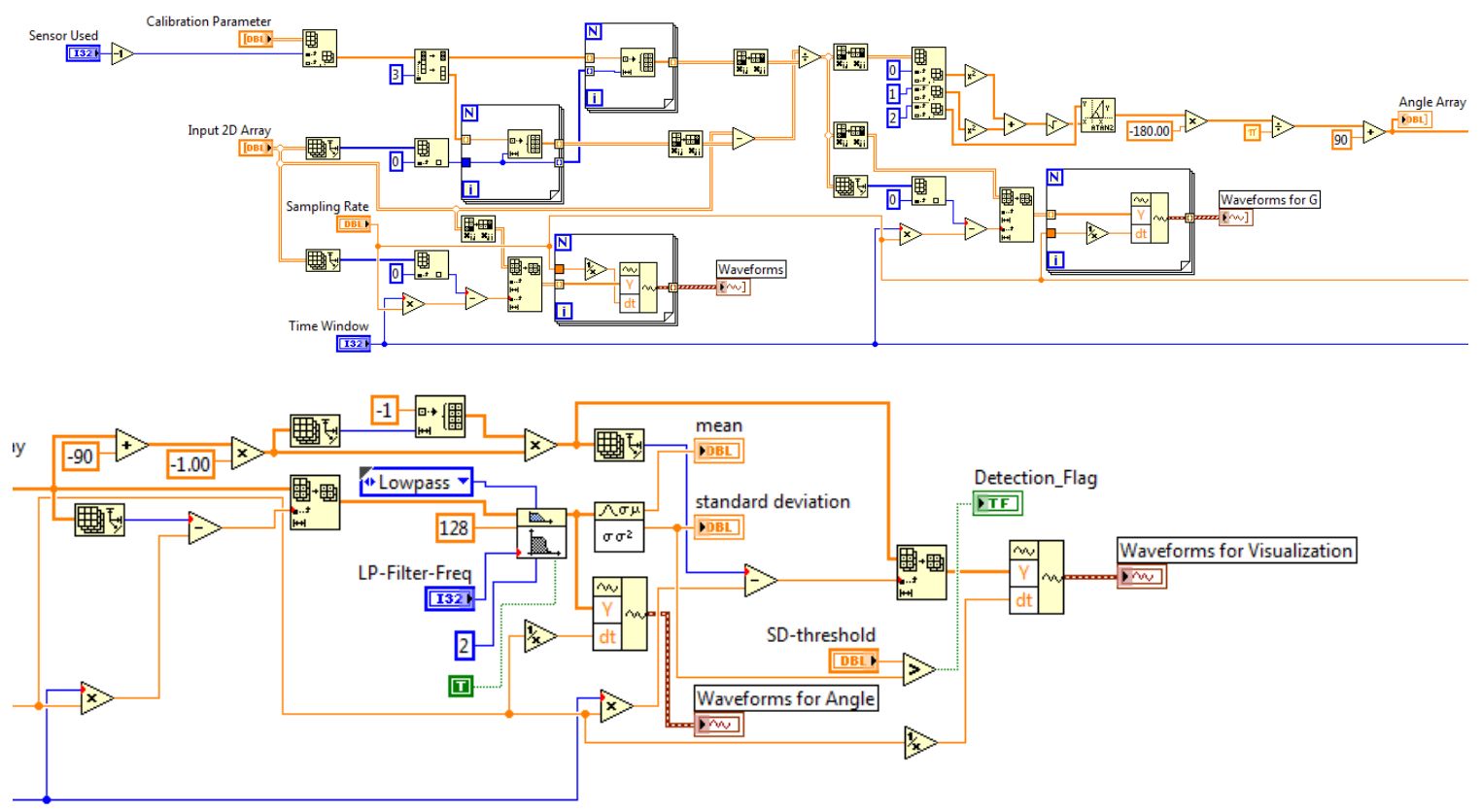


\section{References}

[1] T. T. Chiu, W. Y. Ku, M. H. Lee, W. K. Lee, W. K. Sum, M. P. Wan, C. Y. Wong, C. K. Yuen, "A study on the prevalence of and risk factors for neck pain among university academic staff in Hong Kong", Journal of occupational rehabilitation, vol. 12, no. 2, pp. 77-91, 2002

[2] L. J. Haughie, I. M. Fiebert and K. E. Roach, "Relationship of forward head posture and cervical backward bending to neck pain," Journal of Manual and Manipulative Therapy, vol. 3, pp. 91-97, 1995.

[3] E. Hickey, M. Rondeau, J. Corrente, J. Abysalh and C. Seymour, "Reliability of the cervical range of motion (CROM) device and plumb-line techniques in measuring resting head posture (RHP)," Journal of Manual and Manipulative Therapy, vol. 8, pp. 10-17, 2000.

[4] R. Fejer, K. Kyvik and J. Hartvigsen, "The prevalence of neck pain in the world population: a systematic critical review of the literature," European Spine Journal, vol. 15, pp. 834-848, 2006.

[5] S. Raine and L. Twomey, "Posture of the head, shoulders and thoracic spine in comfortable erect standing," Australian Journal of Physiotherapy, vol. 40, pp. 25$25,1994$.

[6] D. Evick and O. Aksoy, "Relationship between head posture and temporomandibular dysfunction syndrome," Journal of Musculoskeletal Pain 2004, 12, 19-24.

[7] M. Rocabado, "Biomechanical relationship of the cranial, cervical, and hyoid regions." J. Craniomandibular Pract., vol. 1, pp. 61, 1983.

[8] E. Ayub, M. Glasheen-Wray, and S. Kraus, "Head Posture: a case study of the effects on the rest position of the mandible. Journal of Orthopaedic \& Sports Physical Therapy 1984, 5, 179-83. 
[9] D. H. Watson and P. H. Trott, "Cervical headache: an investigation of natural head posture and upper cervical flexor muscle performance," Cephalalgia 1993, $13,272-84$.

[10] R. Cailliet, L. Gross, "The rejuvenation strategy," Doubleday \& Company, Inc. 1987, 52-60.

[11] C. Fernandez-de-las-Penas, C. Alonso-Blanco, M. L. Cuadrado, and J. A. Pareja, "Forward head posture and neck mobility in chronic tension-type headache: a blinded, controlled study," Cephalalgia 2005, 26, 314-19.

[12] W. P. Hanten, S. L. Olson, J. L. Russell, R. M. Lucio, A. H. Campbell, "Total head excursion and resting head posture: normal and patient comparisons," Archives of Physical Medicine and Rehabilitation, vol 81, Issue 1, January 2000, 62-66,

[13] M. W. Darnell, "A proposed chronology of events for forward head posture," $J$ Craniomandib Pract 1983, 1, 50-4.

[14] R. McKenzie, "Treat your own neck. Lower Hutt," New Zealand: Spinal Publications, 1983.

[15] C. Kisner, L. A. Colby, "Therapeutic exercises-foundations and techniques," Philadelphia; EA. Davis, 1988.

[16] S. Kylamarkula, J. Huggare, "Head posture and the morphology of the first cervical vertebra," Eur J Orthod 1985, 7, 1-6.

[17] B. Solow, A. Tallgren, "Head posture and the morphology," Am J Anthropol 1976, 44, 417-36.

[18] H. M. C. Lau, T. T. W. Chiu and T. Lam, "Clinical measurement of craniovertebral angle by electronic head posture instrument: A test of reliability and validity," Manual Therapy., vol. 14, pp. 363, 2009.

[19] B. Valachi and K. Valachi, "Preventing musculoskeletal disorders in clinical dentistry: Strategies to address the mechanisms leading to musculoskeletal disorders," The Journal of the American Dental Association, vol. 134, pp. 1604, 2003.

[20] M. A. Hanson, H. C. Powell, A. T. Barth, K. Ringgenberg, B. H. Calhoun, J. H. Aylor, J. Lach, "Body Area Sensor Networks: Challenges and Opportunities," IEEE Computer, vol.42, no.1, pp.58-65, Jan. 2009

[21] C. H. Willford, C. Kisner, T. M. Gleenn, and L. Sachs, "The interaction of wearing multifocal lenses with head posture and pain," Journal of Orthopedic and Sports Physical Therapy 1996, 23, 194-9. 
[22] C. H. T Yip, T. T. W. Chiu, and A. T. K. Poon, "The relationship between head posture and severity and disability of patients with neck pain," Manual Therapy, vol 13, no. 2, pp. 148-154, 2008

[23] M. Wilmarth and T. Hilliard, "Measuring head posture via the craniovertebral angle," Orthop Phys Ther Pract, vol. 14, pp. 13-15, 2002.

[24] A.T. Barth, M.A. Hanson, H.C. Powell Jr., J. Lach, "TEMPO 3.1: A Body Area Sensor Network Platform for Continuous Movement Assessment," International Conference on Body Sensor Networks, 71-6, 2009

[25] S. A. Ridenour, "Flexible and Efficient Platform for Body Sensor Networks." M.S. thesis U of Virginia, Charlottesville, 2011.

[26] Freescale Semiconductor, “ $\pm 4 \mathrm{~g}, \pm 12 \mathrm{~g}$ Three Axis Low-g Micromachined Accelerometer," MMA7331L Rev 0 datasheet, April 2008, accessed April 2011

[27] I. Frosio, F. Pedersini, and N.A. Borghese, "Autocalibration of MEMS accelerometers," IEEE Transactions on Instrumentation and Measurement, pp. 2034-2041, 2009.

[28] P. Lukowicz, H. Junker, and G. Troster, "Automatic calibration of body worn acceleration sensors," Pervasive Computing, pp. 176-181, 2004.

[29] J. Borenstein, L. Ojeda, and S. Kwanmuang, "Heuristic reduction of gyro drift in IMU-based personnel tracking systems," SPIE Defense, Security + Sensing, Conference 7306A: Optics and Photonics in Global Homeland Security V, pp. 73061H-11, 2009.

[30] A. Krohn, M. Beigl, C. Decker, U. Kochendörfer, P. Robinson, and T. Zimmer, "Inexpensive and Automatic Calibration for Acceleration Sensors", In Proceedings of the Second international conference on Ubiquitous Computing Systems, 2005.

[31] "Tracker - Video Analysis and Modeling Tool" [Online]. Available: http://www.cabrillo.edu/ dbrown/tracker/ 


\section{List of Publications}

[P1] S. Chen, J.S. Brantley, T. Kim, J. Lach, Characterizing and Minimizing Synchronization and Calibration Errors in Inertial Body Sensor Networks, International Conference on Body Area Networks, accepted for publication, 2010

[P2] A.T. Barth, B.C. Bennett, B. Boudaoud, J.S. Brantley, S. Chen, C.L. Cunningham, T. Kim, H.C. Powell, Jr., S.A. Ridenour, J. Lach, Longitudinal High-Fidelity Gait Analysis with Wireless Inertial Body Sensors, Wireless Health, 192-3, 2010

[P3] T. Kim, S. Chen, J. Lach, Detecting and Preventing Forward Head Posture with Wireless Inertial Body Sensor Networks, International Conference on Body Sensor Networks, PP. 125-126, 2011.5

[P4] S. Chen, J.S. Brantley, T. Kim, S.A. Ridenour, J. Lach, Characterizing and Minimizing Sources of Error in Inertial Body Sensor Networks, International Journal of Autonomous and Adaptive Communications Systems, 2013 